\title{
Modernité de la catoptrique de Héron d'Alexandrie
}

\section{Alain Boutot}

\section{OpenEdition}

\section{Journals}

Édition électronique

URL : https://journals.openedition.org/philosant/938

DOI : 10.4000/philosant.938

ISSN : 2648-2789

\section{Éditeur}

Éditions Vrin

\section{Édition imprimée}

Date de publication : 1 novembre 2012

Pagination : 157-196

ISBN : 978-2-7574-0400-3

ISSN : 1634-4561

\section{Référence électronique}

Alain Boutot, « Modernité de la catoptrique de Héron d'Alexandrie », Philosophie antique [En ligne], 12 | 2012, mis en ligne le 01 novembre 2018, consulté le 02 décembre 2022. URL : http:// journals.openedition.org/philosant/938; DOI : https://doi.org/10.4000/philosant.938

Creative Commons - Attribution - Pas d'Utilisation Commerciale - Pas de Modification 4.0 International - CC BY-NC-ND 4.0

https://creativecommons.org/licenses/by-nc-nd/4.0/ 


\title{
MODERNITÉ DE LA CATOPTRIQUE DE HÉRON D'ALEXANDRIE
}

\author{
Alain BOUTOT \\ Université de Bourgogne
}

RÉSUMÉ. Dans sa Catoptrique, Héron d'Alexandrie déduit la loi de la réflexion de la lumière en s'appuyant sur un principe inédit, le principe du plus court chemin. L'article, après avoir retracé les grandes lignes de la démonstration, s'attache à mettre en évidence l'originalité et la fécondité de la méthode utilisée. Le recours à un principe d'extrémalité pour rendre compte des phénomènes lumineux tranche avec l'approche qu'adoptera Ptolémée dans son Optique par exemple, et anticipe par certains côtés l'optique géométrique moderne, dont Héron apparaît comme une sorte de précurseur involontaire. Mais au-delà de l'aspect purement historique, l'article insiste sur la portée épistémologique majeure du principe héronien, et des principes d'extrémalité en général, qui s'inscrivent dans une tradition scientifique bien précise, et toujours actuelle, celle qui privilégie, au rebours de l'expérimentalisme et de l'instrumentalisme, la causalité finale et formelle dans l'explication des phénomènes et promeut en définitive les mathématiques au rang de principe ontologique fondamental. Dans cette perspective, la démarche suivie dans la Catoptrique révèle non seulement sa profonde modernité, mais pourrait bien apparaître comme une des plus emblématiques qui soient.

SUMmARY. In his Catoptrics, Hero of Alexandria infers the law of reflection of light from an original principle, the principle of the shortest path. This paper, after outlining the proof, aims at highlighting the novelty and fertility of Hero's method. By accounting for the phenomena of light by means of a principle of extremality, Hero defines an approach very different from the one adopted e. g. by Ptolemy in his Optics; in some ways this is an anticipation of modern geometrical optics, of which Hero appears as an unintentional forerunner. Outside the purely historical point of view, this paper emphasizes the epistemological significance of Hero's principle and, generally speaking, of principles of extremality. These principles belong to a particular scientific tradition which is still alive: the tradition which favours final and formal causality over experimentalism and instrumentalism, and finally promotes mathematics to the rank of a basic ontological principle. From this point of view, Hero's approach in his Catoptrics not only shows it as utterly modern, but could well be seen as one of the most emblematic ones. 

Héron, mécanicien alexandrin du début de notre ère, a été quelque peu malmené par l'histoire et par les historiens. Son nom et ses écrits ont sombré dans un oubli à peu près total jusqu'au début du siècle précédent, époque à laquelle l'ensemble de son œuvre, ou du moins ce qu'il en reste, a été rééditél. Mais les rares commentateurs qui se sont intéressés à lui ont aussitôt souligné la portée théorique limitée des textes désormais disponibles. Ainsi J.-L Heiberg, historien des mathématiques grecques et éditeur des Stereometrica et du De Mensuris de Héron déclare: « Héron n’est pas un savant, mais un technicien appliqué et un arpenteur. Ce point de vue, que l'on a vainement cherché à récuser, a été exprimé pour la première fois par Hermann Diels : "un simple artisan" (ein reiner Banause) $)^{2}$. Rien ne caractériserait mieux notre auteur que ce passage des Métriques où il explique avoir voulu « rassembler tous les écrits utilisés avant lui et qui lui sont parvenus », ou cet autre de la Balistique, où il se présente comme un vulgarisateur des travaux de ses prédécesseurs qu'il juge trop techniques. Certains, comme l'historienne I. Hammer-Jensen, n'ont pas hésité à le présenter comme un ignorant qui se serait contenté de recopier des travaux qu'il ne comprenait pas toujours. D’une façon générale, Héron apparait sous les traits d'un compilateur, plus ou moins bien informé, mais sans véritable originalité, dont le principal mérite serait de nous fournir une sorte de témoignage sur l'état des sciences et des techniques de son temps ${ }^{3}$.

Ce qui vaut pour l'ensemble de son ouvre vaut bien évidemment aussi pour sa Catoptrique. Celle-ci n'est généralement pas envisagée en ellemême, mais se trouve replacée au sein d'une histoire qui va d'Euclide à Ptolémée, histoire dont Héron aurait été davantage le témoin que l'acteur. Albert Lejeune, par exemple, retrace, dans ses Recherches sur la catoptrique grecque, l'émergence de la méthode expérimentale dans l'optique alexan-

1. Schmidt 1899-1914 (abrégé ci-dessous en Opera).

2. Heiberg 1925, p. 37.

3. Drachmann 1981, plus nuancé dans son jugement, croit pouvoir affirmer qu'il aurait enseigné les mathématiques, la physique, la pneumatique et la mécanique à Alexandrie, et serait à ce titre l'auteur de manuels à l'intention des étudiants ou des hommes de l'art (architectes, ingénieurs, etc.). Cf. Dictionary of Scientific Biography, New York, 1981, t. 5-6, s. $v$. « Hero of Alexandria » p. 311. 
drine de la façon suivante : «On reconnaît aujourd'hui unanimement que les Grecs surent être des philosophes subtils, des mathématiciens géniaux et même de bons observateurs, mais on admet encore généralement qu'ils n’ont jamais dépassé le stade de l'observation généralisée hâtivement. Le traité de Ptolémée nous laisse apercevoir que l'optique et la catoptrique ont évolué dans un sens qui fait prévoir la conception moderne de la physique, et nous atteste que les Alexandrins ont à l'occasion pratiqué déjà fort judicieusement la méthode mathématico-expérimentale 4 . $\gg$ La Catoptrique de Héron porterait à ses yeux la marque de cette évolution. « La présence de Héron parmi les catoptriciens est probablement l'indice d'une influence externe qui est venue renforcer la tendance expérimentale interne de la catoptrique [...] Héron est de la lignée de Ctésibius et de Philon de Byzance, c'est-à-dire de ceux dont on admet généralement qu'ils représentent dans la science grecque la tournure d'esprit la plus voisine de notre physique expérimentale ${ }^{5}$. Jean Itard explique que « les principaux traités hellénistiques et gréco-romains [dans le domaine de l'optique] qui nous ont été conservés sont l'Optique d'Euclide, la Catoptrique de Héron, une Catoptrique d'un pseudo-Euclide (Théon d'Alexandrie?), l'Optique de Ptolémée. De ces quatre ouvrages, les plus remarquables, ajoute-t-il, sont le premier et le dernier $»^{6}$, la contribution de Héron apparaissant donc comme mineure et en tout cas comme secondaire.

Cette présentation des choses ne rend pas justice, nous semble-t-il, à Héron. Il y a en effet, dans sa Catoptrique, une argumentation que les historiens ont certes relevée, mais sans en saisir toute la portée et l'originalité. Héron déduit en effet dans ce traité la loi de la réflexion de la lumière d'un principe géométrique, le principe du plus court chemin. « On peut trouver de l'intérêt, commentent $\mathrm{P}$. Brunet et $\mathrm{A}$. Miéli, à sa démonstration de la propriété que présente le rayon lumineux d'avoir l'angle de réflexion égal à l'angle d'incidence. La base essentielle en est le principe que la lumière suit le chemin le plus court, et la démonstration est faite tant pour les miroirs plans que pour les concaves ou convexes ${ }^{7}$. $\gg$ Cette démonstration n'a pas, à notre sens, qu'un intérêt anecdotique. Elle se distingue fondamentalement, par son esprit, de la démonstration donnée par Ptolémée de la même loi, et est même totalement nouvelle. Elle obligerait même à corriger l'image de constructeur ou d'inventeur d'engins trop souvent accolée à Héron, qui occulte le mathématicien ou le géomètre. Loin d'être l'artisan appliqué que l'on a dit, Héron sait faire preuve d'imagination et même d'audace. Nous

\footnotetext{
4. Lejeune 1957, p. 187-188.

5. Ibid., p. 182.

6. Itard 1966, p. 349.

7. Brunet \& Mieli 1935, p. 498.
} 
voudrions montrer que loin d'être un simple maillon faisant la transition entre Euclide et Ptolémée, notre alexandrin fait signe en réalité par delà Ptolémée vers l'optique géométrique moderne dont il constitue une sorte de précurseur involontaire. Mais auparavant, il nous faut dire quelques mots sur ce qu'on appelle la « question héronienne », celle de savoir qui était Héron, quand il a vécu et ce qu'il a écrit; nous nous pencherons ensuite sur la Catoptrique en nous intéresssant plus particulièrement à la démonstration de la loi de la réflexion; en troisième lieu nous mettrons en évidence l'originalité et la fécondité du principe sur lequel elle repose ; et enfin nous dégagerons le contenu épistémologique de ce principe.

\section{La vie et l'œuvre de Héron}

« En dehors de ses œuvres, affirme un commentateur contemporain, D. R. Hill, nous ne savons rien de Héron, et, jusqu'à une époque très récente, l'époque où il vécut était l'objet d'une controverse ${ }^{8}$. $\gg$ Thomas Henri Martin recense, dans ses Recherches sur la vie et les ouvrages d'Héron d'Alexandrie, pas moins de vingt-et-un personnages ayant porté le nom de Héron dans l'Antiquité. Un seul d'entre eux aurait été mathématicien, celui qui nous intéresse, dont Théon nous apprend qu'il aurait été cordonnier avant de se tourner vers la philosophie. Aucune source littéraire ne le mentionne avant Pappus (300 apr. J.-C.), qui cite ses Mécaniques. Héron cite lui-même Archimède (mort en 212 av. J.-C.), et semble connaître Appollonius. Il aurait donc vécu entre 150 av. J.-C. et 250 apr. J.-C., ce qui laisse malgré tout une marge d'incertitude de quatre siècles. Toutes les époques ou presque ont du reste été proposées pour le situer. Ainsi, Th. H. Martin le fait vivre pendant la première moitié du I ${ }^{\text {er }}$ siècle avant notre ère? B. Carra de Vaux, éditeur et traducteur du manuscrit arabe des Mécaniques ou l'élévateur des corps lourds, le ramène à l'ère chrétienne, et en fait un contemporain de Ptolémée (II ${ }^{\mathrm{e}}$ siècle), en invoquant la présence de nombreux latinismes dans son œuvre, et le fait que Vitruve ( $\mathrm{I}^{\text {er }}$ siècle apr. J.-C.), qui connaît et cite Ctésibius et Philon, ne mentionne jamais Héron. W. Schmidt, l'éditeur allemand, estime au contraire que Héron est antérieur à Ptolémée, car il ignore son évaluation de la circonférence de la Terre. Il le situe au I ${ }^{\text {er }}$ siècle $^{10}$, en se fondant sur la description, à la fin de ses Mécaniques, d'un pressoir d'un genre particulier dont l'utilisation serait postérieure à 55 apr. J.-C. selon Pline. D’autres l'ont placé après Ptolémée, en arguant que ce dernier se servait d'instruments moins perfectionnés que ceux

8. Hill 1988, p. 9.

9. Martin 1854, p. 28.

10. Opera, I, p. XXIV. 
décrits par Héron. La question héronienne semble avoir été tranchée aujourd'hui, depuis qu'O. Neugebauer a relevé, dans un article ${ }^{11}$ paru en 1938, que l'éclipse de lune décrite par Héron au chapitre 35 de sa Dioptrique, éclipse visible à Alexandrie et à Rome, ne pouvait correspondre qu’à celle du 13 mars 62 apr. J.-C. Comme tout porte à croire que Héron a été le contemporain du phénomène, on peut considérer, avec le préfacier de la réédition de la traduction de B. Carra de Vaux, D. R. Hill, que « la controverse sur les dates de Héron ne présente plus aujourd'hui qu'un intérêt historique $\gg^{12}$. Héron d'Alexandrie a donc vécu au $\mathrm{I}^{\mathrm{er}}$ siècle de notre ère.

Qui était-il ? Eh bien d'abord et avant tout un mécanicien. «Par ses inventions et plus encore par ses ouvrages, notamment par son traité de Mécanique, [...] écrit Th. H. Martin, Héron s'était placé au premier rang des mécaniciens grecs $\gg$. Il fait partie de la tradition des ingénieurs qui, depuis le III siècle av. J.-C., à Alexandrie, se sont employés a tirer les applications pratiques de certaines découvertes scientifiques, et dont les plus fameux furent Ctésibius et son disciple Philon de Byzance ${ }^{13}$. La mécanique comportait une partie théorique, consistant en « géométrie, arithmétique, astronomie et physique $\gg^{14}$ et une partie pratique. À ce titre, elle traitait, comme l'explique Proclus, aussi bien de l'équilibre des corps et de la position des centres de gravité que de la fabrication d'engins utiles pour la guerre, du fonctionnement d'appareils à prodiges ou de la construction des sphères ${ }^{15}$. Parmi les écrits de mécanique attribués à Héron et parvenus

11. Neugebauer 1938, p. 3-24.

12. Hill 1988, préface, p. 9 .

13. On peut joindre Vitruve à cette série.

14. Pappus, Collection mathématique, VIII, 1 (p. 1022, 15-17 Hultsch).

15. Commentaires sur le premier livre des Eléments d'Euclide, 41, 3 Friedlein sq. Pappus évoque, dans sa Collection mathématique, les mécaniciens de l'école de Héron, et explique que la mécanique comportait une partie théorique (géométrie, arithmétique, astronomie et physique) et une partie pratique. «Parmi les arts mécaniques, ajoute Pappus, les plus nécessaires, du point de vue des besoins vitaux, sont les suivants : 1) l'art des constructeurs de poulies, que les Anciens appelaient mécaniciens. Grâce à leurs machines, ils utilisent une force moindre pour élever des poids importants dans le sens contraire à leur tendance naturelle ; 2) l'art de ceux qui fabriquent des instruments nécessaires à la guerre, appelés eux aussi mécaniciens. Des projectiles de pierre, de métal et autres sont lancés à grande distance par des catapultes qu'ils fabriquent ; 3) l'art des fabricants de machines proprement dits. L'on peut faire facilement monter de l'eau d'une grande profondeur grâce aux machines hydrauliques qu'ils construisent ; 4) les Anciens appelaient aussi mécaniciens les fabricants de prodiges. Certains ont inventé des appareils pneumatiques, comme Héron dans ses Pneumatiques, d'autres paraissent imiter des êtres vivants à l'aide de tendons et de cordelettes, comme le fait Héron dans ses Automates et dans son ouvrage Sur les balances; d'autres emploient des objets flottants, comme Archimède dans son ouvrage Sur les corps flottants, ou des horloges hydrauliques, comme Héron dans son traité Sur les horloges hydrauliques, qui a une connexion manifeste avec l'étude du gnomon ; 5) on appelle aussi mécaniciens ceux qui 
jusqu'à nous, figurent les Pneumatiques, les Automates, les Belopoiica (les machines de guerre) et les Mécaniques. Le plus connu et le plus long d'entre eux est le premier cité - les Pneumatiques - dont la plus grande partie concerne la description de machines mues par des variations de pression hydrostatique ou aérostatique. Quelques-unes de ces machines ont une finalité utilitaire, comme la pompe à incendie (I, 28) ou l'orgue hydraulique (I, 52), mais la plupart n'ont d'autre ambition que de divertir: des vases truqués versant, selon le cas, du vin ou de l'eau (I, 9), des oiseaux qui sautent et chantent, des trompettes qui sonnent (I, 15-16), des animaux qui boivent quand on leur donne de l'eau (I, 29 sq), des roues de bronze qui, en tournant, nettoient les pieds de ceux qui entrent dans un temple et aspergent les personnes d'eau lustrale (I, 32), un temple dont les portes s'ouvrent et se ferment automatiquement lorsqu'on allume un feu sur l'autel (I, 38), des figurines qui s'animent sous l'effet de la chaleur (II, 3), etc. Le plus connu de ces dispositifs est incontestablement la fameuse machine de Héron ou «éolipile » (II, 11), qui utilise la vapeur d'eau pour mettre en rotation une sphère. Le dispositif est le suivant : au-dessus d'un récipient rempli d'eau, on place une sphère creuse, tournant librement autour d'un axe horizontal, munie de deux tubes recourbés situés aux deux extrémités d'un même diamètre, les courbures étant à angles droits et de sens contraires. Elle est alimentée en vapeur d'eau par un tube, plongé dans le récipient. Sous l'effet de la chaleur, l'eau bout, la vapeur entre dans la sphère, et ressort par les tubes. Héron a réussi à transformer l'énergie calorifique en énergie mécanique. Mais il serait exagéré d'en conclure qu'il aurait anticipé en quelque façon la machine à vapeur, d'abord parce que telle n'était pas son intention, et surtout parce que les problèmes qu'il fallait maîtriser pour obtenir une machine à vapeur efficace étaient totalement hors de portée à son époque. «C'est seulement après un développement long et complexe, explique G. E. R. Lloyd, qu'une machine ayant une puissance supérieure à dix chevaux-vapeur fut finalement construite à la fin du XVIII ${ }^{\mathrm{e}}$ siècle $^{16}$. » Le traité des Automates, qui ressemble beaucoup aux Pneumatiques, était lui aussi bien connu à la Renaissance. Il explique le fonctionnement de représentations théâtrales automatiques, mettant en scène des figurines mues par des systèmes de cordes, de poulies, de poids et de contrepoids. Il décrit ainsi une mise en scène de la Légende de Nauplius, montrant les Grecs, de retour du siège de Troie, en train de construire des navires, prendre la mer au milieu des dauphins, et finalement sombrer après avoir été égarés par les

savent fabriquer des sphères, et construire un modèle du ciel en utilisant le mouvement circulaire uniforme de l'eau » (Collection mathématique, VIII, 2, p. 1024, 12-1026, 4 Hultsch).

16. Lloyd 1993, p. 299. 
feux allumés par Nauplius, alors que le chef de la flotte, Ajax, disparaissait foudroyé par Athéna. Le texte original des Mécaniques, un des principaux ouvrages de Héron, n'est pas parvenu jusqu'à nous. Il en existe cependant une version arabe (du $\mathrm{X}^{\mathrm{e}}$ siècle), éditée et traduite en français par B. Carra de Vaux en 1894 sous le titre Les Mécaniques ou l'élévateur des corps lourds. Le premier livre traite de questions cinématiques, comme le roulement des cercles. Il expose aussi le parallélogramme des mouvements, étudie l'équilibre d'un cylindre sur un plan incliné et présente la théorie du centre de gravité. Le second livre contient la description des cinq machines simples (treuil, levier, moufle, coin et vis) et de leurs combinaisons. Le troisième livre analyse le fonctionnement de machines plus compliquées, comme celles permettant de soulever des fardeaux ou les pressoirs à huile ou à vin. Les Mécaniques contiennent également la description du Baroulkos, machine capable de soulever une charge importante grâce à un système d'engrenages.

Héron n'est pas seulement un mécanicien, c'est aussi un mathématicien, et d'abord un géomètre. Moritz Cantor va même jusqu'à en faire, dans ses Leçons sur l'histoire des mathématiques (1880), le chef d'une école de géomètres, celle des géomètres romains ayant fleuri de César à Trajan. Cette filiation est fausse, puisque Héron est plus tardif que Cantor ne le pensait, mais elle est néanmoins révélatrice de l'importance qu'on peut lui accorder. Sa contribution la plus significative dans ce domaine est un ouvrage en trois livres, les Métriques, retrouvé seulement en 1896 et publié en 1903. La géométrie héronienne est une géodésie ( $\gamma \varepsilon \omega \delta \alpha$ เซi - terme qui désigne le partage ou le sectionnement des terres), et traite des méthodes de mesure des longueurs, des surfaces et des volumes. Héron se montre ici fidèle à la vieille tradition faisant dériver la géométrie de la technique de l'arpentage :

Comme l'antique tradition nous l'enseigne, la plupart [des géomètres] s'appliquaient au mesurage et au partage des terres, et c'est de là que la géométrie a pris son nom. L'invention de ce mesurage a été faite chez les Égyptiens. Car, à cause de la crue du Nil, beaucoup de terrains, habituellement à découvert, disparaissaient par le débordement du fleuve, et il y avait beaucoup de propriétés privées qu'il était impossible à chaque propriétaire de reconnaitre après la retraite des eaux. C'est pourquoi les Égyptiens imaginèrent ce mesurage, qui s'exécute tantôt avec ce qu'on appelle une chaine d'arpentage (oxolviov), tantôt avec un roseau, tantôt avec d'autres instruments. Le mesurage étant donc nécessaire, l'usage s'en propagea chez tous les hommes désireux de s'instruire ${ }^{17}$.

17. Cf. Martin 1854, p. 437. Geometrica, Opera, IV, p. 176-177. Cf. aussi Metrica, Opera, III, p. 2. 
Héron donne dans ses Métriques un certain nombre de règles permettant le calcul du périmètre des figures planes, de l'aire des surfaces cylindriques, côniques ou sphériques, comme du volume de différents solides. Il est ainsi l'auteur d'une fameuse formule, qu'on appelle encore « formule de Héron », exprimant l'aire d'un triangle quelconque en fonction de la longueur de ses trois côtés : si $a, b$ et $c$ désignent la longueur des côtés d'un triangle et si $s$ est le demi-périmètre, l'aire $A$ du triangle vaut : $\sqrt{ } s(s-a)(s-b)(s-$ $c)^{18}$. Il résout, dans le troisième livre, quelques problèmes de division des aires et des volumes selon un rapport donné. Héron a ainsi laissé une méthode d'approximation rationnelle des racines carrées ${ }^{19}$ et cubiques ${ }^{20}$. Il existe d'autres ouvrages géométriques attribués à Héron, qui reprennent largement les Métriques (Geometrica, De Mensuris, Stereometrica), ou consistent simplement en recueil de définitions (Definitiones), mais leur intérêt est moindre.

Héron, le mécanicien et le géomètre, est aussi un physicien, et plus précisément un opticien. Il est l'auteur d'une Dioptrique et surtout d'une Catoptrique. C'est sur cette partie de l'œuvre de Héron que nous voudrions à présent nous arrêter ${ }^{21}$.

18. Metrica I, 8, Opera, III, p. 18. Cf. aussi Geometrica, Opera, IV, p. 248-249: « Méthode pour trouver l'aire de tout triangle. Il s'agit de trouver l'aire de tout triangle donné. Faites ainsi qu'il suit : additionnez ensemble les nombres des trois côtés; prenez la moitié de leur somme; de cette somme retranchez le nombre de chaque côté ; multipliez le reste de la soustraction d'un côté par la moitié de la somme des trois côtés, puis le reste de la soustraction d'un autre côté par le produit de la première multiplication, puis encore le reste de la soustraction du troisième côté par le produit de la seconde multiplication, et extrayez la racine carrée du produit obtenu : ce sera l'aire. » (Trad. Martin 1854, p. 439-440.)

19. Cf. Metrica, I, 8, où Héron donne une valeur aprochée de la racine de 720 . La méthode d'approximation d'extraction de la racine carrée de Héron est originale, et diffère de celle d'Archimède qui se fondait sur l'algorithme d'Euclide de division successive. Pour calculer $\sqrt{ } \mathrm{a}$, Héron écrit $\mathrm{a}=\mathrm{r} 2+\mathrm{b}$, d'où $\sqrt{\mathrm{a}}=\mathrm{r} \sqrt{ }(1+\mathrm{b} / \mathrm{r} 2)=\mathrm{r}+\mathrm{b} / 2 \mathrm{r}=1 / 2(\mathrm{r}+\mathrm{a} / \mathrm{r})$. Ainsi $\sqrt{720}=26,5+1 / 3$. En réitérant le processus (ce que Héron ne fait pas), on obtient une suite qui converge très rapidement vers la racine. Cette méthode a été utilisée par Newton.

20. Cf. Metrica III, 20, Opera, III, p. 178. La méthode de Héron doit être reconstruite à partir d'un seul exemple numérique (racine cubique de 100). Heath 1921 propose, p. 341342 , une conjecture sur la formule d'approximation utilisée.

21. Tous les ouvrages de Héron sont tournés vers les applications pratiques. Cependant, on ne trouve pas chez lui l'idée de transformer la nature, de tourner le cours des choses à notre avantage. Les applications relèvent dans leur immense majorité de ce qu'on peut appeler la physique amusante, ou étonnante. Héron a donc un côté thaumaturge, faiseur et montreur de prodiges, de dispositifs destinés à susciter l'étonnement. C'est donc un mécanicien philosophe, si tant est que l'étonnement soit le propre du philosopher. 


\section{La Catoptrique de Héron et le principe d'extrémalité}

\section{Remarques éditoriales}

Un certain mystère entoure la Catoptrique de Héron, dans la mesure où aucun traité portant ce titre ne nous est parvenu sous son nom. Nous savons qu'il a écrit une catoptrique par le témoignage de Damien, doxographe du $\mathrm{IV}^{\mathrm{e}}$ siècle, disciple ou fils de Héliodore de Larisse, qui affirme,

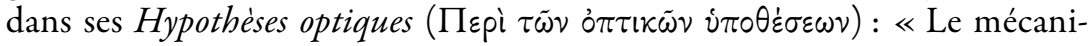
cien Héron a démontré dans sa catoptrique que les droites qui se brisent en formant des angles égaux sont plus courtes que celles qui, ayant les mêmes extrémités, se brisent en formant des angles inégaux ${ }^{22}$. » Ce qu'on appelle aujoud'hui la Catoptrique de Héron est en réalité un texte latin édité sous le nom de Ptolémée : Claudii Ptolemei de Speculis. Nous commencerons par rappeler l'histoire mouvementée de ce texte, et la façon dont il a finalement été attribué à Héron, en nous appuyant principalement sur les Recherches sur la vie et les ouvrages de Héron d'Alexandrie de Th. H. Martin.

Le texte latin Claudii Ptolemei de Speculis a été imprimé à Venise en 1518 dans une collection d'écrits astronomiques, éditée par un médecin physicien, Geronimo Nucerello. Après être tombé dans un oubli à peu près total, ce texte a été exhumé au XIX ${ }^{\mathrm{e}}$ siècle par un commentateur italien, G. B. Venturi, dans ses Commentaires sur l'histoire et la théorie de l'optique (1814). En comparant ce texte avec les livres conservés de l'Optique de Ptolémée, Venturi a remarqué que les propositions qu'il contenait, par leur objet et leur démonstration, ne correspondaient à aucune de celles de l'Optique de l'astronome grec, et notamment à aucune de celles figurant dans les deux livres où Ptolémée traite de la réflexion. Ce texte ne pouvant raisonnablement avoir été rédigé par Ptolémée, Venturi a alors émis l'hypothèse qu'il s'agissait de la catoptrique perdue de Héron, en invoquant trois arguments ${ }^{23}$.

(a) L'auteur du texte latin renvoie, au début, à l'un de ses écrits sur la dioptrique. Or il se trouve que Héron a justement écrit un traité de dioptrique. Précisons que la dioptrique antique ne correspondait nullement à la théorie de la réfraction de la lumière, comme c'est le cas aujourd'hui, mais désignait l'art de mesurer les distances. Elle traitait simplement de l'usage de la $\delta$ io $\pi \tau p \alpha$, instrument de visée ou ligne de mire employée dans la géométrie pratique (géomètres experts) et l'astronomie.

22. Schöne 1897, p. 20.

23. Martin 1871 a montré que l'optique latine, traduite de l'arabe par l'amiral Eugène au XIII siècle, était bien l'optique de Ptolémée (que l'on croyait perdue). Par conséquent, le texte latin édité sous le nom de Ptolémée ne peut pas être de Claude Ptolémée, auteur de l'Almageste. 
(b) Ensuite, la thèse attribuée par Damien à Héron dans ses Hypothèses optiques, selon laquelle le rayon lumineux suit le chemin le plus court, ne se trouve ni chez Euclide, ni chez Ptolémée, mais bien dans le Claudii Ptolemei de Speculis.

(c) Enfin tout débouche, dans ce texte, sur des applications pratiques, utilitaires et surtout amusantes, comme c'est justement le cas dans les ouvrages de Héron.

L'hypothèse de Venturi a été reprise par Th. H. Martin, ainsi que par l'éditeur allemand, W. Schmidt, qui tient « pour définitivement assuré que nous avons dans le Ptolemeus de Speculis la catoptrique de Héron, quoique sous une forme très abrégée et corrompue $\gg^{24}$. Th. H. Martin précise que cette traduction latine n'a pas été faite sur une traduction arabe, à la différence de l'Optique latine de Ptolémée, mais sur un texte grec, comme le montre la présence de nombreux hellénismes ( $\varepsilon \Uparrow \delta \omega \lambda \circ \nu / \pi \circ \lambda \nu \theta \varepsilon \dot{\varepsilon} \omega \rho \circ / \eta \dot{\mu l}$

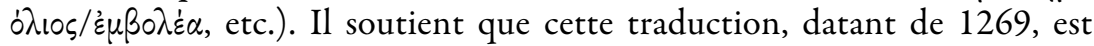
l'œuvre de Guillaume de Mœrbeke, moine « connu comme traducteur de divers ouvrages d'Aristote, de Galien, de Proclus et de Simplicius » ${ }^{25}$, et auquel Vitellion avait dédié son Optique de 1274 après avoir utilisé sa traduction $^{26}$. Cette conjecture lui paraît d'autant plus vraisemblable que « le style de cette traduction offre une ressemblance déplorable avec celui des autres traductions faites par Guillaume de Mœrbeke ${ }^{27}$.

La traduction latine est non seulement peu fiable, mais aussi, ce qui est sans doute plus gênant, fragmentaire. La faute n'en incombe pas au traducteur seul, car le texte grec, à en croire Th. H. Martin, devait être déjà passablement corrompu. Au terme de cette brève enquête, on peut donc reconstituer les différentes phases de la transmission de notre texte de la façon suivante : un abrégé grec de la Catoptrique de Héron a été rédigé au cours de l'un des premiers siècles de notre ère. Cet abrégé, de mauvaise qualité, est resté pendant longtemps anonyme, avant d'être attribué, par une fausse conjecture, à Ptolémée ${ }^{28}$. Au XIII ${ }^{\mathrm{e}}$ siècle, G. de Mœrbeke l'a traduit en latin, le texte grec s'étant ensuite perdu. La traduction de G. de Mœrbeke,

24. Mechanica et Catoptrica, Opera, II, p. 306. Bien que ces trois raisons en faveur de l'attribution du texte à Héron ne soient pas contraignantes, elle rendent cependant la chose vraisemblable. Rose a indiqué que la référence à la musique platonicienne des sphères au début de l'écrit pouvait soulever quelques doutes. Néanmoins, on peut observer que Straton, une des sources de Héron dans le Proemium des Pneumatiques, avait élaboré une sorte de théorie ondulatoire pour l'acoustique et l'optique. La relation entre acoustique et optique dans notre écrit pourrait donc renvoyer à Straton, ce qui lève du même coup l'objection.

25. Martin 1854, p. 63.

26. Selon Martin 1854, p. 70.

27. Ibid.

28. Cf. ibid. p. 86 
publiée en 1518 au milieu d'autres textes dans une collection d'écrits astronomiques, « a été citée vaguement, selon Th. H. Martin, par plusieurs écrivains du XVI ${ }^{\mathrm{e}}$ et du XVII ${ }^{\mathrm{e}}$ siècle, puis elle est tombée dans l'oubli, et elle n'a été mentionnée dans aucune bibliographie, dans aucune histoire de la littérature grecque, dans aucune histoire, soit des sciences mathématiques ou physiques en général, soit de l'optique en particulier $\gg^{29}$.

\section{Le contenu de la catoptrique}

Après ces remarques éditoriales, venons-en au contenu même de l'ouvrage de Héron. Nous allons en donner une présentation d'ensemble, avant de nous arrêter plus longuement sur le principe que le mécanicien met au fondement des lois de la réflexion.

Dans l'édition de W.Schmidt, la Catoptrique est divisée en dix-huit chapitres, plus ou moins étendus, consacrés chacun, sauf exception, à la démonstration d'un théorème particulier ou à la résolution d'un problème spécifique. Après un chapitre introductif (chapitre I), le traité s'articule en deux grandes parties, qui ne sont pas distinguées en tant que telles par l'éditeur : une partie théorique constituée par les chapitres II à $\mathrm{X}$ où sont énoncés et démontrés une série de dix théorèmes, et une partie pratique, regroupant les chapitres XI à XVIII où Héron entreprend de résoudre une série de huit problèmes.

Dans l'introduction, Héron commence par rappeler que, selon Platon, « il y a deux sens qui nous conduisent à la sagesse, l'ouïe et la vue $\gg^{30}$, et qu'il convient donc de les étudier tous les deux. Après quelques considérations sur la musique et l'harmonie des sphères célestes, il en vient à la science de la vue, qu'il divise en trois parties : (1) l'optique, qui est la théorie de la vision proprement dite ; (2) la dioptrique, qui, comme on l'a dit, n'est pas l'étude de la réfraction, mais traite de la visée optique au moyen d'un instrument permettant des relevés topographiques, la dioptre ; (3) la catoptrique, qui est la théorie des miroirs ( $\kappa \dot{\alpha} \tau 0 \pi \tau \rho \circ \nu)$ et de la réflexion. Il poursuit de la façon suivante :

Nos prédécesseurs ont écrit sur l'optique d'une manière satisfaisante, et notamment Aristote. Nous-même avons traité ailleurs de la dioptrique avec

29. Ibid. p. 56.

30. Catoptrique, Opera, II, p. 316. Dans le Phédon, Platon place en effet la vue et l'ouïe au dessus des autres sens: «Est-ce que quelque vérité est fournie aux hommes par la vue aussi bien que par l'ouie, ou bien, là-dessus au moins, en est-il comme les poètes même nous le ressassent sans trêve, et n'entendons-nous, ne voyons-nous rien exactement ? Pourtant, si parmi les sensations corporelles celles-là sont sans exactitude et incertaines, on ne saurait attendre mieux des autres, qui toutes en effet sont, je pense, inférieures à celles-là. » (65b1-6, trad. Robin 1926.) 
l'étendue qui nous a paru convenable. Mais il nous a semblé que la catoptrique était aussi digne d'étude, et offrait d'admirables spéculations. Car c'est par elle qu'on peut réaliser des miroirs où la droite paraît à droite et la gauche à gauche, alors que les miroirs ordinaires font paraître le contraire et contrefont la nature. On peut aussi construire des miroirs où l'on se voit de dos, renversé, la tête en bas, avec trois yeux et deux nez, ou bien avec le visage décomposé, comme dans une profonde douleur. La catoptrique ne permet pas seulement d'obtenir des images de ce genre, mais elle a aussi des applications utiles. En effet qui ne trouverait utile de pouvoir observer les habitants de la maison d'à côté, de voir combien ils sont et ce qu'il font ? Ou comment ne trouverait-on pas merveilleux de voir, de jour comme de nuit, les heures au moyen d'images apparaissant dans un miroir, de sorte qu'il y ait autant d'images que d'heures ? N'est-il pas merveilleux aussi de ne voir dans un miroir ni soi-même ni personne d'autre, mais seulement ce que d'autres voudront? Puisque cette science (negotium) existe, je pense qu'il est de mon devoir d'honorer l'héritage de mes prédécesseurs en l'exposant, afin de n'omettre aucune partie de ma tâche ${ }^{31}$.

La Catoptrique de Héron repose sur une théorie particulière de la vision, qui n'est pas propre à notre auteur, la théorie des rayons visuels : l'œil émet des rayons qui vont rencontrer les objets, soit directement (vision directe) soit indirectement (vision réfléchie). Cette théorie dite émissive, ou théorie du feu visuel, est sans doute d'origine pythagoricienne. Ainsi pour Alcméon $\left(\mathrm{VI}^{\mathrm{e}}-\mathrm{V}^{\mathrm{e}}\right.$ siècle), « la vision s'effectue à travers l'eau qui baigne les yeux : qu'ils contiennent du feu, c'est une évidence : la preuve est qu'un choc provoque des étincelles $\gg^{32}$. Cette théorie se maintiendra dans toute l'Antiquité. On la retrouve, avec des variantes, chez Empédocle $\left(V^{e} \text { siècle }\right)^{33}$, qui compare l'œil à une lanterne, Platon ${ }^{34}$, Euclide, Ptolémée, Galien ${ }^{35}$ (II siècle) et enfin Théon d'Alexandrie (IV ${ }^{\mathrm{e}}$ siècle). Dans cette théorie, qu'Aristote juge du

31. Catoptrica, Opera, II, p. 318-320.

32. Selon le témoignage de Théophraste, De Sensibus, $26=24$ A 5 DK.

33. Cf. Aristote, De la sensation et des sensibles, 437b23, et le commentaire d'Alexandre d'Aphrodise, Commentaire sur le traité de la sensation d'Aristote : « Empédocle compare la projection de la lumière hors des yeux à la lumière <provenant> des chandelles. De même en effet que quelqu'un qui se propose de sortir la nuit se prépare une chandelle et l'introduit dans une lanterne [...], ainsi, déclare-t-il, le feu enfermé dans les membranes est enveloppé par de fines peaux qui préservent le feu des assauts nuisibles de l'extérieur, et ne permettent pas de causer du dommage à la pupille, tandis que l'élément le plus subtil peut sortir de l'œil » (31 B 84 DK, t. I, p. 341, 13-20).

34. Cf. Resp. VI, 508b3-4 : « De tous les organes des sens, l'œil est celui qui tient le plus du soleil » (trad. É. Chambry 1933); Tim. 45b6-9: « [Les dieux] ont fait en sorte que le feu pur qui réside au-dedans de nous et qui est frère du feu extérieur s'écoulât au travers des yeux de façon subtile et continue. » (trad. Rivaud 1925.)

35. Cf. De Usu Partium, X, 12, 815-817. 
reste absurde ${ }^{36}$, la vision s'effectue sur le modèle du toucher. L'œil palpe pour ainsi dire ce qu'il voit. Ses rayons constituent un organe éphémère permettant, selon la remarque de Gérard Simon, de «sentir hors de soi $\gg^{37}$. On sait que ce n'est qu'à partir du $X^{\mathrm{e}}$ siècle, avec Alhazen, mathématicien et physicien cairote, que les choses s'inverseront, et que la lumière, au lieu de sortir de l'œil, y pénétrera pour y imprimer des images. La métaphore de la lanterne sera alors remplacée par celle la chambre noire, comme c'est le cas chez Képler qui, dans ses Compléments à Vitellion (1604), conçoit l'œil sur le modèle d'une chambre obscure, avec un orifice qui serait la pupille, un diaphragme qui serait l'iris, un objectif convergent, qui serait le cristallin et un écran où se forme l'image, qui serait la rétine ${ }^{38}$. C'est cette métaphore, reprise par Descartes dans sa Dioptrique, que nous utilisons encore aujourd'hui, sous une forme sans doute plus élaborée. Pour Héron, les choses se présentaient de manière différente : la lumière va de l'œil vers l'objet, et non de l'objet vers l'œil. G. Simon ${ }^{39}$ fait remarquer à juste titre qu'entre l'optique antique et la nôtre, il n'y a pas seulement une inversion du sens de propagation de la lumière, mais un changement d'objet : alors que l'optique moderne est surtout préoccupée d'établir les lois de la propagation du rayon lumineux, l'optique antique s'attache essentiellement à déterminer le positionnement des images. Il n'en reste pas moins que ce positionnement s'effectue suivant des théorèmes qui, bien qu'ayant été établis à partir d'une hypothèse différente de la nôtre, restent encore valables aujourd'hui, compte tenu de ce phénomène tout à fait remarquable, qu'on appelle le retour inverse de la lumière. Ce sont ces théorèmes que Héron expose dans la première partie de sa Catoptrique, théorèmes qui constituent la base et la justification théorique des dispositifs optiques qu'il étudie dans la seconde partie.

Le premier théorème de Héron pose que les rayons visuels vont de l'œil à l'objet en ligne droite ${ }^{40}$. Héron le prouve en invoquant un principe fondamental : la tendance de la nature à suivre le plus court chemin. Héron commence par appliquer ce principe, que nous qualifierons de principe d'extré-

36. Cf. De la sensation et des sensibles, 438a25: « Il est complètement absurde de soutenir que l'œil voit au moyen de quelque chose qui en sort et que le rayon visuel s'étend jusqu'aux astres, ou que, après avoir parcouru une certaine distance, il fusionne, comme des auteurs le prétendent, avec quelque autre chose qui sort de l'objet. » Pour Aristote, la vision correspond à l'actualisation du diaphane.

37. Simon 1988, p. 36.

38. Descartes reprend cette métaphore pour laquelle il fournit un schéma dans sa Dioptrique (1637).

39. Simon 1988, p. 25.

40. Nous reprenons dans ce qui suit l'excellent résumé de la Catoptrique donné par Martin 1854. 
malité, non pas aux rayons visuels, mais aux objets lancés avec force, comme les flèches tirées par un arc. Ces objets tendent à suivre le chemin le plus court. Or, et c'est là le second théorème, les rayons visuels sont émis avec une vitesse infinie : la preuve en est que «lorsque nous levons les yeux, nous voyons immédiatement les étoiles, bien que leur éloignement soit pour ainsi dire infini $\gg^{41}$. De la conjonction de ces deux propositions, il résulte que les rayons visuels se meuvent en suivant le chemin le plus court, c'est-à-dire en ligne droite. Cette conclusion présuppose une certaine définition de la droite, comme plus court chemin entre deux points, définition qui ne va nullement de soi, puisque Euclide définissait la droite autrement, à savoir comme la ligne «qui repose également sur ses points ${ }^{42}$. Or c'est bien la définition géodésique de la droite, d'origine archimédienne, que Héron retient dans ses Définitions ${ }^{43}$. Dans le troisième théorème, Héron explique pourquoi les surfaces polies réfléchissent les rayons visuels, alors que les surfaces non polies ou les corps transparents les laissent pénétrer voire même traverser. Le quatrième théorème établit l'égalité des angles d'incidence et de réflexion pour les miroirs plans. Héron le démontre au moyen, nous y reviendrons, du principe fondamental du plus court chemin. Dans le cinquième théorème il généralise la loi de l'égalité des angles d'incidence et de réflexion aux miroirs convexes en utilisant le même principe. Dans le sixième théorème, il montre qu'un miroir plan contient un point dont l'occultation empêche l'image d'être vue. Le septième théorème établit que les rayons issus d'un même point et réfléchis par un miroir plan ne sont ni convergents ni parallèles. Le huitième prouve la même proposition pour les miroirs convexes. Le neuvième théorème établit que les rayons visuels émis par un œil situé au centre d'un miroir concave sont tous réfléchis vers l'œil. Héron prétend ainsi prouver qu'un œil placé dans cette situation ne verra rien d'autre que lui-même, ce qui est en fait inexact. Le dixième théorème affirme que les rayons visuels émis par un œil situé à la périphérie d'un miroir concave se coupent. Héron aurait pu en conclure, ce que fera plus tard Vitellion, qu'un miroir concave peut donner deux images différentes d'un même objet. Comme certains l'ont fait remarquer ${ }^{44}$, ces

41. Catoptrica, Opera, II, p. 322.

42. Éléments, I, déf. 4.

43. Definitiones, Opera, IV, p. 16. «Étant donné deux points, la droite est la plus courte des lignes ayant ces points pour extrémités. »

44. Cf. Martin 1854, p. 67 ; Lejeune 1957, p. 138-142. Le neuvième correspond au vingt-quatrième de la Catoptrique d'Euclide, et le dixième au cinquième. D'après Th. $\mathrm{H}$. Martin, Héron aurait reproduit la Catoptrique d'Euclide; d'après A. Lejeune, la Catoptrique dite d'Euclide serait en réalité l'œuvre de Théon d'Alexandrie, et ce dernier se serait inspiré de Héron. 
deux derniers théorèmes se retrouvent presque textuellement dans la $\mathrm{Ca}$ toptrique dite d'Euclide.

Ces théorèmes sont destinés, en principe tout au moins, à servir à la résolution d'un certain nombre de problèmes pratiques ou techniques, que Héron énonce dans la seconde partie de son ouvrage. Il s'agit à chaque fois de trouver un dispositif adéquat, constitué d'un ou de plusieurs miroirs plans, convexes ou concaves, permettant d'obtenir l'effet optique souhaité. Ces problèmes relèvent pour l'essentiel de ce que Th. H. Martin appelle la catoptrique amusante. Nous nous contenterons de les énumérer, sans entrer dans le détail de chacun d'eux. Les deux premiers problèmes consistent à construire des miroirs dextres, c'est-à-dire faisant paraître la droite à droite. Héron décrit deux miroirs de ce genre, le premier cylindricoconcave, le second formé de deux parties courbes juxtaposées (en $« S »)$, l'une concave et l'autre convexe. Le troisième problème est de construire un miroir polythéore, c'est-à-dire renvoyant plusieurs images. La solution est un ensemble de deux miroirs plans rectangulaires superposés, articulés autour d'un axe horizontal, formant un angle variable. Le quatrième problème est de construire ce que le traducteur latin appelle un speculum

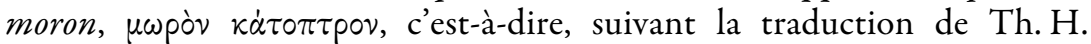
Martin, un miroir nigaud. Ce qualificatif exprimerait, d'après ce dernier, « la mine allongée de ceux qui s'y regardent $»^{45}$. Le speculum moron est un miroir cylindrique convexe. Le cinquième problème est de construire un miroir dit théâtral. Le miroir théâtral est un ensemble de petits miroirs plans verticaux, disposés en demi-cercle sur les côtés d'un polygone régulier. Le spectateur, placé au centre de ce demi-cercle, verra son image se répéter dans chacun des miroirs. Il aura ainsi devant lui, suivant l'expression de Vitellion, « comme un chœur de danse formé par toutes ces images ${ }^{46}$. Le sixième problème est de trouver un dispositif permettant de se voir de dos. La solution est un ensemble de deux miroirs plans formant un angle droit placés en hauteur au-dessus du spectateur, leur surface étant orientée vers le sol. Le spectateur situé à la verticale de l'angle formé par les miroirs verra son image par double réflexion dans le miroir qu'il regardera. Il se verra ainsi par derrière, en l'air et incliné, et ajoute Héron, «pensera voler ${ }^{47}$. Le septième problème réalise la promesse de l'introduction de faire voir dans un miroir placé dans une maison ce qui se passe à l'extérieur, dans la rue. La solution consiste à installer sous la partie supérieure d'une fenêtre donnant sur la rue un miroir plan horizontal. Celui qui est à l'intérieur de la maison pourra ainsi voir les passants à l'extérieur. Héron ne semble pas

45. Martin 1854, p. 75.

46. Cf. Martin 1854, p. 75.

47. Catoptrica, Opera, II, p. 352. 
avoir vu que son dispositif était en réalité à double tranchant, puisque les passants pourront tout aussi bien voir celui qui les observe. Le huitième problème est presque identique au cinquième problème, celui du miroir théâtral: il s'agit de construire un miroir renvoyant à un spectateur la même image de lui-même de plusieurs endroits. La solution est le miroir polygonal, ensemble de plusieurs miroirs plans disposés autour du spectateur et situés à égale distance de lui. Le neuvième et dernier problème s'énonce ainsi : construire un miroir où le spectateur ne se voie pas luimême et ne voie personne d'autre, mais seulement l'image que quelqu'un d'autre a voulu lui faire voir. La solution consiste à disposer, face au spectateur, un miroir plan incliné lui renvoyant l'image d'un objet caché, image elle-même réfléchie par un autre miroir, parallèle au premier, et lui aussi caché. Héron explique que ce système peut être installé dans un temple, ce qui permet de faire apparaître la statue du dieu, sans que le dieu lui-même ne soit visible. Comme le relève Th. $\mathrm{H}$. Martin à la suite de Venturi, « une seule des promesses du préambule ne se trouve point réalisée dans la suite de l'ouvrage : celle de montrer comment on peut faire voir nuit et jour les heures au moyen de fantômes apparaissant dans un miroir $\gg^{48}$. La solution est cependant, selon Th. H. Martin, facile à deviner : elle repose sur l'utilisation d'une horloge hydraulique indiquant l'heure au moyen de statuettes apparaissant par une ouverture. Il est possible que Héron, dans son ouvrage perdu Les horloges hydrauliques, ait imaginé un dispositif de ce genre. Et, comme l'explique Th. H. Martin, « il était facile de cacher une horloge de cette espèce, éclairée pendant la nuit, de telle sorte que la statuette de chaque heure apparût dans un miroir, ainsi qu'il est expliqué dans le neuvième problème de la Catoptrique $\gg{ }^{49}$. On peut penser que l'ouvrage original de Héron décrivait cette solution et s'achevait donc par une application pratique du dernier problème traité.

\section{La démonstration héronienne de la loi de la réflexion}

Héron invoque le principe du plus court chemin à deux reprises dans sa Catoptrique : pour démontrer la propagation en ligne droite du rayon visuel d'une part, et pour démontrer l'égalité des angles d'incidence et de réflexion d'autre part. Nous avons déjà indiqué les grandes lignes de la première démonstration, celle de la rectilinéarité du rayon. Nous allons maintenant expliciter la seconde, celle concernant le phénomène de la réflexion. Cette démonstration, donnée par Héron, dont l'existence est simplement mentionnée par Damien dans ses Hypothèses optiques ${ }^{50}$, a été reproduite,

48. Martin 1854, p. 81.

49. Ibid.p. 82.

50. Schöne 1897, chap. 14, p. 21. 
sous une forme légèrement différente, par Olympiodore ( $\mathrm{VI}^{\mathrm{e}}$ siècle) dans son commentaire des Météorologiques d'Aristote ${ }^{51}$.

Considérons donc, avec Héron, un miroir plan $A B$, et deux points $\Gamma$ et $\Delta$ situés du même côté du miroir. L'œil, source du rayon visuel, est placé en $\Gamma$, l'objet vu en $\Delta$. Partant du principe que le rayon visuel doit suivre le plus court chemin pour aller de $\Gamma$ à $\Delta$, Héron entend prouver l'égalité des angles d'incidence et de réflexion :

Qu'ainsi donc les rayons tombant sur les corps polis sont réfléchis, cela a été, pensons-nous, suffisamment démontré. Mais qu'ils font, en se réfléchissant, des angles égaux, aussi bien sur des miroirs plans que sur des sphériques, nous allons le démontrer de la même façon, à savoir par la rapidité de l'incidence et de la réflexion. Il est en effet nécessaire d'entreprendre de le prouver en utilisant de nouveau les droites les plus courtes ${ }^{52}$.

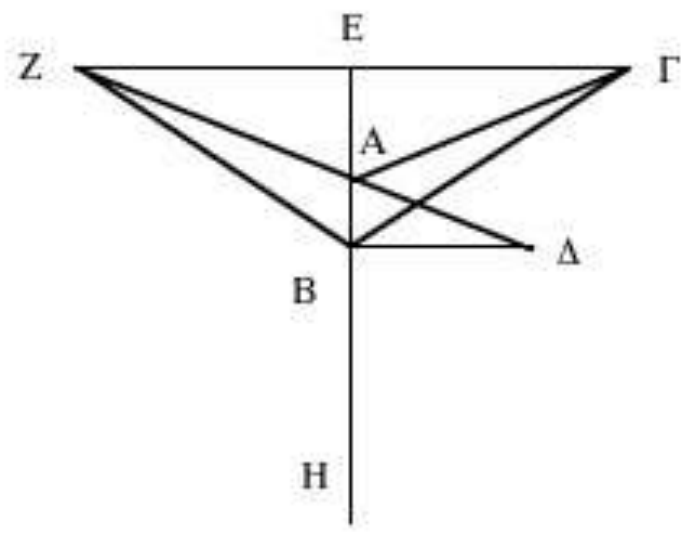

À y regarder de plus près, le raisonnement de Héron, tel qu'on vient de le présenter, est totalement incompréhensible. En effet, il est clair que le plus court chemin pour aller de $\Gamma$ à $\Delta$ est tout simplement la droite joignant $\Gamma$ à $\Delta$, et ne passe donc pas par le miroir. Le phénomène de la réflexion contredit de manière flagrante le principe du plus court chemin. En réalité Héron ne tire pas la loi du seul principe, mais du principe associé à une condition supplémentaire : que le rayon visuel doive, à un moment de son

51. Olympiodori in Aristotelis Meteora Commentaria, 212-213 Stüve. La démonstration figure dans un commentaire du passage suivant des Météores : « Parlons du halo et de l'arcen-ciel, et indiquons leur nature et la cause qui les produit. » (III, 2, 371b18.)

52. Catoptrica, Opera, II, p. 324, 16-22. 
parcours, frapper le miroir. Héron affirme en réalité que de tous les rayons visuels venant de $\Gamma$, rencontrant le miroir et repartant vers $\Delta$, le rayon visuel effectivement réfléchi est le plus court.

Je dis donc, explique-t-il, que de tous les rayons incidents et réfléchis en un même point (i.e. $\Delta$ ), aussi bien sur les miroirs plans que sur les sphériques, les plus courts sont ceux qui forment des angles égaux, et qu'en ce cas, c'està-dire avec les angles égaux, la réflexion se fait conformément à la raison ${ }^{53}$.

Voyons donc la démonstration. Elle comporte une légère distorsion par rapport à l'intention initiale dans la mesure où Héron ne déduit pas directement l'égalité des angles du principe de minimum, mais montre que si l'égalité des angles est réalisée, le principe est satisfait, et qu'il l'est seulement dans ce cas. Soit le rayon visuel allant de $\Gamma$ à $\Delta$ incident au miroir plan $\mathrm{AB}$ au point $\mathrm{A}$ et réfléchi suivant deux angles égaux. Considérons un autre rayon, reliant lui aussi $\Gamma$ à $\Delta$, également incident au miroir mais en un autre point $B$. Héron montre que cet autre rayon est plus long que le précédent, c'est-à-dire que $\Gamma \mathrm{B} \Delta$ est plus long que $\Gamma A \Delta$. Pour ce faire, il abaisse la perpendiculaire de $\Gamma$ sur le miroir, et la prolonge. Elle coupe la droite $\Delta \mathrm{A}$ en un point situé de l'autre côté du miroir : Z. Les angles BA $\Delta$ et ZAE sont égaux, étant opposés par le sommet. Puisque, par hypothèse, l'angle BA $\Delta$ (angle de réflexion) est égal à l'angle EAГ (angle d'incidence), l'angle ZAE est donc égal à l'angle EAГ. Les deux triangles rectangles ZAE et EA sont par conséquent égaux, ayant une base commune et deux angles à la base égaux. Par conséquent $\mathrm{ZA}$ est égal à $\mathrm{A} \Gamma$. On montre de la même façon (en considérant cette fois l'égalité des triangles $\mathrm{ZAB}$ et $\mathrm{BA} \Gamma$ qui ont deux angles égaux : $Z A B$ et $\Gamma A B$, un côté commun : $A B$ et deux côtés égaux : $Z A$ et $\Gamma A$ ) que $\mathrm{ZB}$ est égal à $\mathrm{B} \Gamma$. Considérons à présent le triangle $\mathrm{Z} \Delta \mathrm{B}$ : le côté $\mathrm{Z} \Delta$ est inférieur à la somme des deux autres côtés, $\mathrm{ZB}+\mathrm{B} \Delta$. Or $\mathrm{Z} \Delta=\mathrm{ZA}+\mathrm{A} \Delta$ $=\Gamma \mathrm{A}+\mathrm{A} \Delta$ (le trajet passant par $\mathrm{A}$ ), et $\mathrm{ZB}+\mathrm{B} \Delta=\Gamma \mathrm{B}+\mathrm{B} \Delta$ (le trajet passant par $B)$. Par conséquent, $\Gamma A+A \Delta$ est plus petit que $\Gamma B+B \Delta$. Ce raisonnement de géométrie élémentaire prouve que le rayon visuel réfléchi à angles égaux est plus court que le rayon réfléchi à angles inégaux (en $\mathrm{B}$ ), et que la réflexion obéit à un principe de minimum.

La démonstration de Héron ne correspond pas tout à fait, nous l'avons dit, à celle annoncée au début du chapitre et n'est pas, à ce point de vue, absolument satisfaisante. Héron montre bien que si la réflexion se fait à angles égaux, elle obéit au principe de minimum, mais il ne montre pas que la réflexion se fait à angles égaux parce qu'elle obéit à un principe de minimum. Il substitue en quelque sorte le principe à la conséquence. En fait cette distorsion n'est pas très gênante; on peut en effet penser que Héron 
ne procède pas de manière progressive mais régressive : il ne part pas $\mathrm{du}$ principe pour aller à la conséquence, mais de la conséquence pour remonter au principe qui la fonde. Le principe d'extrémalité constitue la raison du phénomène de la réflexion, et c'est bien ainsi que les commentateurs, du reste, l'ont compris. On peut sans difficulté, du reste, modifier la démonstration de Héron pour la mettre sous la forme désirée ${ }^{54}$.

Héron donne une démonstration parallèle pour les miroirs convexes, mais il serait trop long de l'exposer. Il ne donne pas la démonstration pour les miroirs concaves, contrairement à ce qu'il avait promis. Cette absence n'est pas due à un simple oubli, mais s'explique par une difficulté liée à ce genre de miroirs, qui semblent en effet mettre en défaut le principe du plus court chemin.

\section{Originalité de la démonstration héronienne}

La démonstration héronienne de la loi fondamentale de la réflexion est beaucoup plus originale qu'on pourrait le croire au premier abord. Héron est en effet le seul opticien de l'Antiquité à avoir établi l'égalité des angles d'incidence et de réflexion en utilisant le principe du plus court chemin. Ce principe n'est invoqué ni par ses prédécesseurs, c'est-à-dire Euclide et Archimède, ni par ses successeurs, c'est-à-dire Ptolémée et Théon d'Alexandrie. Ceux-ci connaissent bien sûr eux aussi la loi de la réflexion, mais ils l'établissent de manière fondamentalement différente.

Ainsi, dans la Catoptrique dite d'Euclide, dont l'authenticité est du reste contestée (elle serait l'œuvre en réalité de Théon d'Alexandrie, compilateur du IV ${ }^{e}$ siècle), la loi de l'égalité des angles d'incidence et de réflexion apparaît comme un théorème, c'est-à-dire comme une proposition démontrée à partir d'hypothèses. C'est même le premier des trente théorèmes que compte le traité : «Les rayons visuels sont réfléchis sous des angles égaux par les miroirs plans, convexes et concaves ${ }^{55}$. $\gg$ Ce théorème est établi à partir de la définition (öpos) suivante qui est en réalité un postulat:

Qu'un miroir étant posé sur un plan, il y a proportion telle que la hauteur de celui qui regarde est à la hauteur établie à angles droits sur le plan comme

54. La démonstration rigoureuse de la loi de la réflexion est donnée par exemple par R. Feynman, dans son cours de Mécanique, t. 2, Paris, 1979, p. 5. Considérons, sur la figure de la p. 174 , le trajet $\Gamma B \Delta$. Le problème est de trouver le point $\mathrm{B}$ tel que le trajet $\Gamma \mathrm{B} \Delta$ soit le plus petit possible. Soit le point $Z$, symétrique de $\Gamma$ par rapport au miroir. Le miroir étant la médiatrice de $\Gamma Z$, on a $\Gamma \mathrm{B}=\mathrm{ZB}$. Donc le trajet $\Gamma \mathrm{B}+\mathrm{B} \Delta$ est égal à $\mathrm{ZB}+\mathrm{B} \Delta$. On voit immédiatement que $\mathrm{ZB}+\mathrm{B} \Delta$ sera minimal si $\mathrm{Z}, \mathrm{B}$ et $\Delta$ sont alignés, et donc lorsque le point $\mathrm{B}$ sera en $\mathrm{A}$. Mais $\mathrm{Z}$, $\mathrm{A}$ et $\Delta$ étant alignés, l'angle $\mathrm{BA} \Delta$ est égal à l'angle $\mathrm{ZAE}$ (angles opposés par le sommet), et donc à l'angle EA bien l'égalité des angles d'incidence et de réflexion.

55. Euclide, L'Optique et la catoptrique, trad. fr. par Ver Eecke 1938, p. 100. 
la droite menée entre le miroir et celui qui regarde est à la droite menée entre le miroir et la hauteur établie à angles droits ${ }^{56}$.

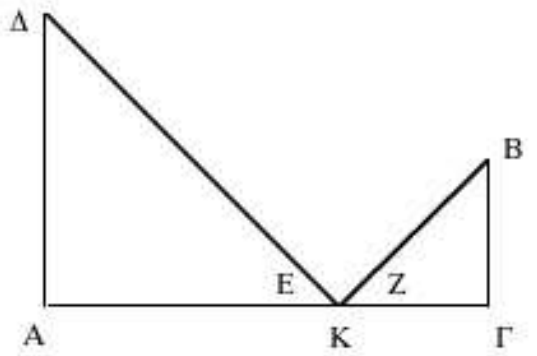

Dans la figure ci-dessus, le miroir est représenté par $\mathrm{A} \Gamma$, l'œil est situé en $\mathrm{B}$ et l'objet vu en $\Delta$. Le postulat affirme que $\mathrm{B} \Gamma / \Delta \mathrm{A}=\mathrm{K} \Gamma / \mathrm{KA}$. Les deux triangles $\triangle \mathrm{AK}$ et $\mathrm{B} \Gamma \mathrm{K}$ sont semblables, et par conséquent l'angle d'incidence $Z$ est égal à l'angle de réflexion $\mathrm{E}$.

D’après Albert Lejeune, la loi de la réflexion se présentait de manière sensiblement différente dans la Catoptrique originale d'Euclide, aujourd'hui perdue. Elle n'y apparaissait pas comme un théorème, mais comme un postulat. C'est du moins ce que semble indiquer la démonstration de la proposition XIX de l'Optique, dans laquelle Euclide invoque la loi de la façon suivante: «Puisque le rayon $\Gamma \mathrm{H}$ est incident et le rayon HA réfléchi, ils

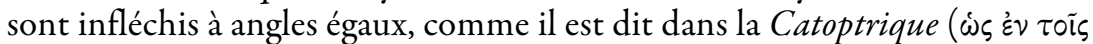

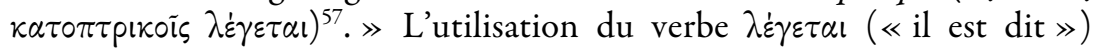
indiquerait, selon A. Lejeune, que la loi figurait dans la Catoptrique parmi les propositions indémontrées. A. Lejeune fait remarquer que Théon, dans sa recension de l'Optique d'Euclide, remplace $\lambda \dot{\varepsilon} \gamma \varepsilon \tau \alpha l$ par $\delta \varepsilon i \kappa \nu v \tau \alpha l^{58}$, « insistant ainsi sur le fait qu'il s'agit désormais d'une démonstration géométrique $\gg^{59}$. Théon penserait avoir introduit une innovation importante en réussissant à établir déductivement ce qu'Euclide se voyait contraint de demander à l'expérience. Quoi qu'il en soit, il est clair que ni Euclide ni Théon ne font intervenir à aucun moment un quelconque principe d'extrémalité.

Archimède, au III $^{\mathrm{e}}$ siècle av. J.-C., avait lui aussi écrit une Catoptrique, mais qui n'est pas non plus parvenue jusqu'à nous. Cependant une note figurant en marge du premier théorème de la Catoptrique dite d'Euclide

56. Ibid. p. 99.

57. Ibid. p. 62.

58. Cf. ibid. p. 67.

59. Lejeune 1957, p. 62. 
rapporte un raisonnement du mathématicien, destiné à prouver précisément l'égalité des angles d'incidence et de réflexion.

Archimède dit que l'angle $Z$ est égal ou plus petit ou plus grand que l'angle E. Soit d'abord $\mathrm{Z}$ plus grand que E : E est alors plus petit. Supposons inversement que l'œil soit en $\Delta$ et que la réflexion ait lieu en sens contraire, de l'œil vers l'objet B. L'angle E sera alors plus grand que l'angle Z. Mais il était aussi plus petit : ce qui est absurde ${ }^{60}$.

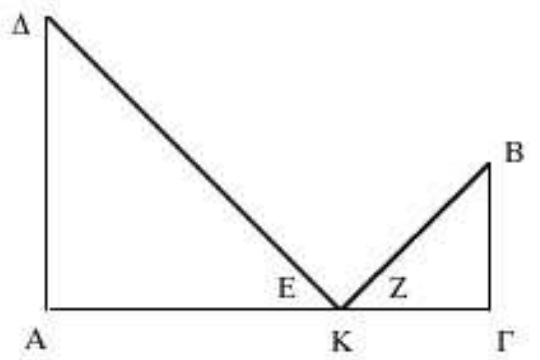

Archimède établit la loi par un raisonnement par l'absurde. Si l'angle d'incidence est plus grand que l'angle de réflexion, alors, en changeant les positions respectives de l'œil et de l'objet, cet angle deviendra plus petit, ce qui est impossible. L'hypothèse initiale (inégalité des angles) est donc fausse, et sa négation (égalité des angles) vraie. En réalité, ce raisonnement n'est concluant qu'à la condition d'admettre une hypothèse supplémentaire, qu'Archimède ne mentionne pas, celle du retour inverse de la lumière. Le rayon lumineux, ou visuel pour Archimède, suit le même trajet pour aller de B à $\Delta$ que de $\Delta$ à $\mathrm{B}$. Le silence d'Archimède s'explique sans doute par le fait qu'il considérait ce phénomène comme bien connu. Il l'aurait même établi expérimentalement, selon A. Lejeune, grâce à une méthode de visée, méthode dont on trouve une trace chez Héron ${ }^{61}$ et Ptolémée ${ }^{62}$. Quoi qu'il en soit, la démonstration archimédienne de la loi de la réflexion, aussi élégante soit-elle, ne fait intervenir nulle part, elle non plus, un quelconque principe d'extrémalité.

Ptolémée, dans son Optique - que nous ne connaissons que par une traduction latine médiévale (due à l'émir Eugène de Sicile) d'une traduction arabe - range la loi de la réflexion parmi les trois «principes (principia)

60. Euclide, L'Optique et la catoptrique, Ver Eecke 1938, p. 100, note 2. Nous avons retenu, en la modifiant, la traduction de Lejeune 1957, p. 51.

61. Cf. Catoptrica, chap. VI, Opera, II, p. 330.

62. Lejeune 1956, III, 4, p. 89. 
nécessaires à la science des miroirs $\gg^{63}$. Le premier de ces principes affirme que «les objets vus apparaissent sur le prolongement du rayon visuel $\gg^{64}$ incident, le deuxième, que « chaque point de [l'objet] qui est vu à travers les miroirs apparaît sur la normale qui tombe de l'objet sur la surface spéculaire et la perce ${ }^{65}$, et le troisième, que « la position du rayon brisé entre la pupille et le miroir et entre le miroir et l'objet est telle que chacun de ses deux tronçons se rencontrent au point sur lequel s'opère la brisure et forment avec la normale élevée de ce point sur le miroir des angles égaux $»^{66}$. Ces principes reposent tous sur «l'hypothèse fondamentale de l'optique suivant laquelle l'œil émet un cône de rayons rectilignes qui vont percevoir dans l'espace les objets $»^{67}$. Ptolémée met en relation le troisième principe, l'égalité des angles d'incidence et de réflexion, avec les lois générales de la mécanique.

Le rapport d'égalité des angles de la réflexion, explique-t-il, répond à un processus naturel. En effet, les obstacles s'opposent à peine aux projectiles quant ils sont tangents à leur trajectoire, mais les obstacles situés sur cette trajectoire s'y opposent fortement. C'est pourquoi lorsqu'un obstacle s'oppose à un projectile de plein fouet et fermement, il interrompt la trajectoire et s'oppose à elle [...]. Ainsi les parois rejettent les balles qui tombent sur elles à angles droits. Les autres [scil. les obstacles tangents] ne font nullement obstacle, à la façon dont la poignée des arcs ne s'oppose pas aux flèches. Il faut comprendre de la même façon toute espèce de mobile et savoir qu'ils se comportent ainsi ${ }^{68}$.

Ptolémée fait appel ici au parallélogramme des mouvements. Le mouvement d'un mobile est la somme d'un mouvement horizontal (tangent à l'obstacle) et d'un mouvement vertical, perpendiculaire. Après rebond, le mouvement tangent est conservé, alors que le mouvement vertical est inversé. Les rayons visuels obéissent à cette loi de la mécanique, qui sera du reste utilisée par Descartes dans sa Dioptrique. Un rayon visuel normal au miroir est réfléchi vers lui-même. Un rayon oblique conserve, après réflexion, « la position réalisée dans l'interception parfaite, c'est-à-dire que l'angle formé par la droite d'interception parfaite avec la droite d'interception partielle [est] égal à celui formé par la droite inverse d'interception parfaite

63. Ibid. p. 88. Le livre I manque, le livre II traite du visible, les livres III et IV des miroirs et le livre $\mathrm{V}$ de la réfraction.

64. Ibid. p. 88.

65. Ibid.

66. Ibid.

67. Lejeune 1957, p. 34.

68. Lejeune 1956, III, 19, p. 98. 
avec celle de l'interception partielle $»^{69}$. Cela veut dire que le rayon réfléchi conserve «sa direction relative par rapport au rayon normal et au rayon rasant $\gg^{70}$. Ptolémée ne se contente pas de ces considérations théoriques, mais décrit un certain nombre d'expériences destinées à prouver les principes de la catoptrique. Ainsi, en ce qui concerne la loi de la réflexion, il imagine, pour prouver l'égalité des angles, une situation dans laquelle deux observateurs se regardent l'un l'autre dans un même miroir. L'expérience montre que leurs rayons visuels visent le même point du miroir, car ils ne se voient plus si on obture ce point. Leurs rayons visuels suivent donc des trajets réciproques, d'où il résulte «que la réflexion se produit à angles égaux $\gg^{71}$. Ptolémée propose ici en quelque sorte une traduction expérimentale du raisonnement d'Archimède. Mais pas plus que son illustre prédécesseur, il n'utilise à aucun moment un quelconque principe d'extrémalité.

L'originalité de la démarche de Héron est donc indéniable. L'utilisation du principe du plus court chemin, comme fondement de la loi de la réflexion, a été non seulement inédite, mais elle est restée sans lendemain, du moins dans l'Antiquité. Car après avoir sombré dans un oubli à peu près total, et ce pour des raisons qui ne tiennent pas seulement aux contingences de l'histoire, le principe de Héron va refaire surface à l'époque moderne et acquérir par la suite une portée quasi-universelle. Nous allons retracer l'histoire de sa redécouverte - qui est aussi celle de ses métamorphoses - à travers trois étapes essentielles : l'extension du principe de Héron à la réfraction par Fermat, sa transposition à la mécanique dans le principe de moindre action, et la conciliation du principe de moindre temps et du principe de moindre action dans la mécanique ondulatoire.

\section{Modernité du principe de Héron}

\section{Le principe de moindre temps}

Le principe de Héron revient sur le devant de la scène scientifique au $\mathrm{XVII}^{\mathrm{e}}$ siècle avec Pierre Fermat, qui l'étend au cas de la réfraction. « Originellement, affirme le physicien américain Richard Feynman, Héron d'Alex-

69. Ibid. III, 20, p. 99.

70. Lejeune 1957, p. 35.

71. Cf. Lejeune 1956, III, 6. Le passage est le suivant : « Ceci se confirme encore quand la position des yeux est telle que chacun voit l'autre au même endroit, ce qui se produit lorsque de l'un et l'autre œil en même temps la vision se fixe sur un seul et même point du miroir. Sinon aucun des deux yeux n'a de l'autre une image propre et cela signifie que les rayons visuels suivent des trajets réciproques. Il en résulte que la réflexion se produit à angles égaux ». A. Lejeune suggère que Ptolémée décrit dans ces lignes la vision réciproque des deux yeux d'un même individu. 
andrie énonça que la lumière se déplace d'une manière telle qu'elle va vers le miroir puis vers l'autre point en empruntant la distance la plus courte possible [...]. Ce fut cela qui suggéra à Fermat que peut-être la réfraction devait opérer sur une base semblable $\gg^{72}$. Fermat ne connaissait pas Héron, mais a découvert le principe de l'alexandrin, et donc son propre principe, par l'intermédiaire d'un de ses correspondants, Marin Cureau de la Chambre, médecin du roi et auteur d'un traité sur la Lumière, paru une première fois en 1634, puis réédité, sous une forme considérablement augmentée, en mars 1657. Dans ce traité, Cureau de la Chambre aborde la question de la réflexion de la lumière, et se demande pourquoi elle se fait à angles égaux. Il ne propose pas lui-même de nouvelle explication, mais se contente de passer en revue celles qui ont été proposées, qui sont de deux types. « Il y a de si grands esprits, écrit-il, qui se sont exercés sur cette question qu'il est bien difficile non seulement d'ajouter quelque chose à ce qu'ils ont dit, mais encore de prendre parti dans la diversité de leurs sentiments. Car ils ont formé deux opinions là-dessus, qui sont toutes deux si vraisemblables qu'il y a de la peine à juger quelle peut être la meilleure. En effet, se peut-il rien dire de plus conforme à la raison que lorsqu'ils assurent que l'égalité des angles dans la réflexion se fait selon les lois que la nature garde en tous les mouvements. Car comme celle-ci emploie en toutes ses actions les moyens les plus courts, elle fait mouvoir les choses par l'espace le plus court : d'où vient que tous les corps vont tout droit à leur centre et que les pesants descendent en bas et les légers montent en droites lignes, parce que ces lignes sont les plus courtes de toutes. De sorte qu'il faut sur cette règle que la réflexion se fasse par les lignes qui soient les plus courtes. Or il est assuré que ces lignes font des angles égaux et que si par impossible les angles n'étaient pas égaux, ces lignes ne seraient pas les plus courtes ${ }^{73}$. » Cureau de la Chambre ne fait que reproduire ici l'opinion de Héron, qu'il ne cite pas, et pour cause. Même s'il ne mentionne pas ses sources, il est très probable qu'il se réfère en réalité à Vitellion, dont l'Optique avait été publiée pour la première fois en 1572 avec les œuvres d'Alhazen par Friedrich Risner dans l'Opticae Thesaurus ${ }^{74}$, avant d'être popularisée par Kepler, dans ses Compléments à Vitellion de 1604 . Or dans son Optique, Vitellion, qui ne prétend pas faire œuvre originale, reproduit l'ensemble ou presque de la Catoptrique de Héron, la quasi-totalité de ses théorèmes et l'intrégralité de ses problèmes, sans les attribuer explicitement à Héron lui-même, mais seulement,

72. R. Feynman, dans Feynman, Leighton \& Sands, t. 2, p. 5.

73. Cureau de la Chambre 1657, p. 311.

74. Risner, 1572. 
conformément à la tradition, à Ptolémée. En ce qui concerne la question particulière de la réflexion, qu'il aborde dans le livre V, Vitellion part du principe selon lequel la nature ne fait rien en vain et « agit toujours en suivant les lignes les plus courtes » $(\mathrm{V}$, théorème 5$)$, puis affirme, après Héron, que les lignes les plus courtes sont celles qui font des angles égaux (V, théorème 18). Lorsque Cureau de la Chambre reprend cette thèse dans son traité, il la doit donc indirectement au mécanicien. L'explication de la réflexion par le principe du plus court chemin soulève cependant, aux yeux de Cureau de la Chambre, deux objections majeures. Ce principe est mis en défaut, en effet, dans deux cas : celui des miroirs concaves (que Héron, en effet, laisse de côté) et celui de la réfraction. « Si la nature faisait les mouvements par les lignes les plus courtes, affirme Cureau de la Chambre, il faudrait qu'elles se trouvassent dans la réfraction; cependant celles qui contiennent l'angle de l'incidence et celui de la réfraction sont plus longues que pas une autre qui se tire d'une de leurs extrémités à l'autre [...]. Ces objections, poursuit-il, qui ont paru invicibles aux philosophes modernes, les ont engagés à chercher une autre raison de cette égalité d'angles et ils ont cru qu'il fallait chercher dans le mouvement des corps, qui est plus facile à connaître que celui de la lumière, la cause de cet effet pour l'appliquer aux rayons. Ils disent donc que quand un corps tombe obliquement sur un plan, il a un mouvement composé de deux autres, à savoir de celui qui est perpendiculaire et de celui qui est parallèle au $\operatorname{plan}^{75}$. » À travers les philosophes modernes, Cureau de la Chambre vise en réalité Descartes, qui donne en effet dans sa Dioptrique de 1637 une explication mécanique de l'égalité d'angles fondée sur la composition des mouvements, explication déjà proposée par Ptolémée. À quoi Cureau de la Chambre objecte que la lumière est une, que ses rayons n'ont qu'une sorte de mouvement, et ne peuvent avoir des inclinations diverses. Il se range en définitive à la première opinion, celle de Héron, qui « lui paraît la plus raisonnable », en dépit des objections qu'elle soulève, et qu'il tente, du reste, de lever. Dans une lettre d'août 1657, Fermat, à qui Cureau de la Chambre venait d'adresser son livre, félicite celui-ci d'avoir préféré justifier l'égalité des angles d'incidence et de réflexion par un principe d'extrémalité : « Je reconnais [...] avec vous la vérité de ce principe que la nature agit toujours selon les voies les plus courtes. Vous en déduisez très bien l'égalité des angles de réflexion et d'incidence, et l'objection de ceux qui disent que les deux lignes qui conduisent la vue ou la lumière dans le miroir concave sont très souvent les plus longues, 
n'est point considérable $\gg^{76}$, ce que Fermat montre en effet. Puisque ce principe « a servi à la réflexion, poursuit le mathématicien, pourrons-nous en tirer quelque usage pour la réfraction? Il me semble que la chose est aisée et qu'un peu de géométrie nous pourra tirer d'affaire $\gg^{77}$. La réfraction obéit, comme la réflexion, à un principe de minimum. Cependant, il ne peut plus s'agir à l'évidence d'un minimum de distance, mais selon Fermat, d'un minimum de résistance. La lumière réfractée suit un chemin tel que la somme des résistances opposées par les milieux traversés soit minimale. Dans la correspondance de 1657, Fermat se contente d'indiquer dans quelle direction chercher la solution. Il ne résoudra ce problème qu'en 1661, en identifiant la résistance d'un milieu au temps mis par la lumière pour le traverser. Dans la réfraction, la lumière suit le trajet correspondant au temps le plus bref (principe de moindre temps). Si la lumière réfractée se rapproche de la normale en passant d'un milieu rare à un milieu dense, c'est parce qu'elle retarde en quelque sorte le plus longtemps possible le moment d'entrer dans le milieu offrant le plus de résistance. Cela suppose bien sûr que la lumière se propage moins vite dans les milieux denses que dans les milieux rares, contrairement à ce que pensait Descartes.

Fermat n'a pu opérer cette extension du principe de Héron à la réfraction que parce qu'il était en possession, et ceci dès 1638 , d'une méthode mathématique très efficace de résolution des problèmes d'extremums. Cette méthode, dite de maximis et minimis, affirme qu'une fonction atteint un extremum lorsque sa première variation, ou, comme on dit aujourd'hui, sa dérivée première s'annule. Voyons comment Fermat l'utilise ${ }^{78}$.

76. Tannery \& Henry 1894 , p. 354-355.

77. Ibid. p. 355.

78. Nous donnons une version simplifiée de la démonstration de Fermat, exposée dans Analyse pour les réfractions (Tannery \& Henry 1896, p. 149-151) et Synthèse pour les réfractions (ibid.p. 151-156). 
Considérons donc deux points $A$ et $B$ situés de part et d'autre de la surface de séparation de deux milieux 1 et 2 d'indices différents, le rayon lumineux allant de $A$ à $B$.

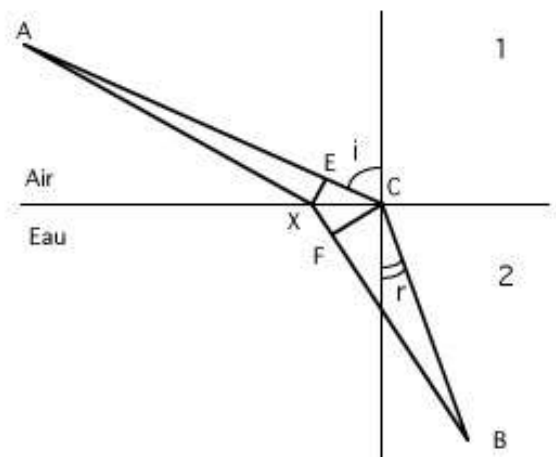

Le problème est de déterminer le point de la surface où le rayon se brise. Ce point doit, d'après le principe de Fermat, minimiser le temps. D'après la méthode de maximis et minimis, il minimise le temps si la première variation du temps est nulle. Cette première variation est égale à la différence entre le temps mis par la lumière sur le trajet $\mathrm{ACB}$ et le temps mis sur un trajet voisin, soit $\mathrm{AXB}$. En désignant par $v_{1}$ et par $v_{2}$ la vitesse de la lumière dans les milieux 1 et 2 , la différence des temps vaut $\mathrm{XF} / v_{2}-\mathrm{EC} / v_{1}$. Celle-ci doit être nulle, ce qui impose $\mathrm{XF} / v_{2}=\mathrm{EC} / v_{1}$. Or $\mathrm{XF}=\mathrm{XC} \sin r$ et $\mathrm{EC}$ $=\mathrm{XC} \sin i, r$ et $i$ désignant les angles des rayons réfracté et incident avec la normale. La condition de minimum implique donc que $n_{1} \sin i=n_{2} \sin r, n_{1}$ et $n_{2}$ désignant les indices de réfraction ${ }^{79}$ des milieux 1 et 2 .

En se fondant sur le principe du moindre temps, Fermat retrouve donc la loi des sinus que Descartes avait établie en partant, remarquons-le, d'une hypothèse exactement inverse, c'est-à-dire en supposant que la lumière allait plus vite dans les milieux denses que dans les milieux rares, plus vite dans l'eau que dans l'air. « Le prix de mon travail, déclare Fermat à son correspondant, Cureau de la Chambre, a été le plus extraordinaire, le plus imprévu et le plus heureux qui fut jamais [...] Car... j’ai trouvé que mon principe donnait exactement et précisément la même proportion des réfractions que M. Descartes a établie. J'ai été si surpris d'un évènement si peu attendu, que j’ai peine à revenir de mon étonnement ${ }^{80}$. $» \ll$ Est-il possible, écrit-il ailleurs, d'arriver sans paralogisme à une même vérité par deux

79. L'indice de réfraction $n$ d'un milieu est égal à $c / v, c$ étant la vitesse de la lumière dans le vide, et $v$ sa vitesse dans le milieu considéré

80. Fermat à Cureau de la Chambre, lettre du ler janvier 1662, Tannery \& Henry 1894, p. 457. 
voies absolument opposées, c'est une question que nous laissons à examiner aux géomètres assez subtils pour la résoudre rigoureusement. ${ }^{81} \gg$

Le principe de Fermat n'infirme pas, en réalité, le principe de Héron mais le généralise. Il le fait apparaître comme un cas particulier obtenu lorsque les milieux traversés sont identiques. En effet, dans la réflexion, la lumière suit aussi bien le trajet le plus court que le trajet le plus bref, puisque sa vitesse est constante. L'unité des deux principes a été soulignée par Leibniz, du reste, qui les fait dépendre, dans son Unique principe d'optique, de catoptrique et de dioptrique (1682), d'un principe métaphysique, celui de la voie la plus facile. «La principale hypothèse commune à ces sciences, d'après laquelle la direction des rayons de toute la lumière est géométriquement déterminée, peut être fondée ainsi : la lumière rayonnant vers un point à éclairer parvient par le chemin le plus facile de tous ${ }^{82}$. » L'application de ce principe en optique simple entraîne la propagation rectiligne de la lumière, en catoptrique l'égalité des angles d'incidence et de réflexion et en dioptrique la loi des sinus. Leibniz renvoie sur les deux premiers points aux anciens, et plus précisément, pour ce qui concerne la catoptrique à Ptolémée, c'est-à-dire en réalité à Héron: «Ptolémée et d'autres anciens se servirent de cette démonstration (i.e. la démonstration de la loi de la réflexion par le principe du plus court chemin), et tantôt elle apparaît ailleurs, tantôt chez Héliodore de Larisse ${ }^{83}$. » Un peu plus tard, dans le Discours de Métaphysique (1686), Leibniz explique que « cherchant la voie la plus aisée pour conduire un rayon donné à un autre point donné par la réflexion d'un point donné (supposant que c'est un dessein de la nature), ils [les anciens] ont trouvé l'égalité des angles d'incidence et de réflexion, comme l'on peut voir dans un petit traité d'Héliodore de Larisse, et ailleurs. Ce que M. Snellius, comme je crois, et après lui (sans rien savoir de lui), M. Fermat ont appliqué plus ingénieusement à la réfraction $\gg^{84}$. Le petit traité dont parle Leibniz n'est autre en réalité que celui de Damien, disciple d'Héliodore de Larisse (Hypothèses optiques), traité édité à Paris en 1657 par le physicien danois Erasme Bartholin ${ }^{85}$. La postérité n’a pas retenu

81. Synthèse pour les réfractions (Tannery \& Henry 1896, p. 152).

82. Unicum opticæ, catoptricæ et dioptricæ principium, Acta eruditorum, juin 1682, trad. fr. Peyroux 1985, p. 10.

83. Ibid.

84. Discours de métaphysique, $\$ 22$.

85. Erasme Bartholin devait découvrir, du reste, un peu plus tard, en 1669 , le phénomène de la double réfraction. Leibniz évoque également Ptolémée, c'est-à-dire Héron dans un opuscule de 1697, Essai anagogique sur la recherche des causes: « Il se trouve que les anciens et Ptolémée entre autres s'étaient déjà servis de cette Hypothèse du chemin le plus aisé du rayon qui tombe sur un plan, pour rendre raison de l'égalité des angles d'incidence et de réflexion, qui est le fondement de la Catoptrique. Et c'est par cette même hypothèse que 
le nom de Leibniz, cependant, mais celui de Fermat pour être associé au principe fondamental de l'optique géométrique moderne. En effet le principe leibnizien du chemin le plus aisé ou le plus déterminé est en réalité bien moins déterminant, c'est-à-dire beaucoup plus vague, et donc plus difficilement utilisable que le principe dit de Fermat, qui ne fait intervenir qu'une grandeur objective et mesurable : le temps ${ }^{86}$.

\section{Le principe de moindre action}

Après avoir été étendu à la réfraction par Fermat, le principe d'extrémalité de Héron a été transposé à la mécanique, où il a pris une signification et une ampleur nouvelles. Laplace, dans son Exposition du système du monde de 1796, retrace ainsi les étapes ayant conduit à cette nouvelle utilisation du principe :

Plusieurs philosophes frappés de l'ordre qui règne dans la nature, et de la fécondité de ses moyens dans la production des phénomènes, ont pensé qu'elle parvient toujours à son but par les voies les plus simples. En étendant cette manière de voir à la mécanique, ils ont cherché l'économie que la nature avait eue pour objet dans l'emploi des forces et du temps. Ptolémée [i.e. Héron d'Alexandrie] avait reconnu que la lumière réfléchie parvient d'un point à un autre par le chemin le plus court, et par conséquent, dans le moins de temps possible, en supposant la vitesse du rayon lumineux toujours la même. Fermat, l'un des plus beaux génies dont la France s'honore, généralisa ce principe, en l'étendant à la réfraction de la lumière [...]. Il trouva conformément à l'expérience, que les sinus d'incidence et de réfraction devaient être dans un rapport constant, plus grand que l'unité. La manière heureuse dont Newton a déduit ce rapport de l'attraction des milieux fit voir à Maupertuis que la vitesse de la lumière augmente dans les milieux diaphanes [i.e. en passant du vide au diaphane] et qu'ainsi ce n'est point, comme Fermat le prétendait, la somme des quotients des espaces décrits dans le vide et dans le milieu, et divisés par les vitesses correspondantes, mais la somme des produits de ces quantités qui doit être minimum. Euler étendit cette supposition aux mouvements variables à chaque instant [en mettant le principe sous une forme intégrale]; et il prouva par divers exemples que, parmi toutes les courbes qu'un corps peut décrire en allant d'un point à un autre, il choisit toujours celle dans laquelle l'intégrale du produit de sa masse par sa vitesse et par l'élément de la courbe, est un minimum ${ }^{87}$.

M. Fermat rendit raison de la loi de la réfraction selon les sinus » (Tentamen anagogicum, dans Gerhardt 1890, p. 274).

86. Cf. les critiques formulées par Euler, dans ses Mémoires de l'Académie de Berlin, 1751, à l'encontre du principe leibnizien, rapportées par Janet 1882, p. 711.

87. Exposition du système du monde (1796), livre III, chap. 2, Serres 1984, p. 204-205. 
La transposition du principe d'extrémalité en mécanique a été opérée par le mathématicien Maupertuis dès 1744. Maupertuis était un tenant de la théorie émissive et corpusculaire de la lumière, et pensait qu'elle allait plus vite dans les milieux denses que dans les milieux rares, sa vitesse étant proportionnelle à la densité des milieux (ou à leur indice de réfraction). Voulant maintenir cette relation de proportionnalité, Maupertuis imagine de substituer au principe de moindre temps de Fermat un autre principe d'extrémalité, celui de moindre action.

J'ai pensé, dit-il, que la lumière, lorsqu'elle passe d'un milieu dans un autre, abandonnant déjà le chemin le plus court, qui est celui de la ligne droite, pouvait bien aussi ne pas suivre celui du temps le plus prompt; en effet, quelle préférence devrait-il y avoir ici du temps sur l'espace? La lumière ne pouvant aller à la fois par le chemin le plus court et par celui du temps le plus prompt, pourquoi irait-elle plutôt par l'un de ces chemins que par l'autre? Aussi ne suit-elle aucun des deux; elle prend une route qui a un avantage plus réel [...] C'est la quantité d'action qui est la vraie dépense de la Nature ${ }^{88}$.

La Nature n'économise ni l'espace ni le temps, mais l'action, celle-ci étant définie comme le produit de la masse par la vitesse et par la distance. De ce principe, on déduit immédiatement la loi de la réfraction de Descartes, comme cela peut se vérifier facilement, du moins si l'on admet que la lumière se propage plus vite dans l'eau que dans l'air. Cette supposition s'étant avérée fausse ${ }^{89}$, le principe de Maupertuis ne s'applique pas en optique, mais demeure au contraire parfaitement valable en mécanique, domaine où Maupertuis l'avait étendu d'une manière apparemment gratuite. « D'une erreur optique, qu'on ne saurait lui reprocher car elle était celle de son temps, commentent Dugas et Costabel, celle de Newton contre Huyghens, [Maupertuis] fait par chance une vérité mécanique ${ }^{90}$. Un point matériel va d'un point à un autre en minimisant l'action. Le principe de moindre action de Maupertuis, après avoir été mis sous une forme intégrale par Euler, sera reformulé par Lagrange puis par Hamilton ${ }^{91}$, qui le mettront au fondement de la mécanique analytique ou rationnelle.

\section{La mécanique ondulatoire}

Le principe de moindre temps de Fermat, et le principe de moindre action de Maupertuis peuvent être considérés, l'un et l'autre, comme des

88. Maupertuis 1753 , p. $98-99$.

89. L’expérience de Fizeau et Foucault, destinée à prouver que la lumière va moins vite dans l'eau que dans l'air, date de 1850.

90. Allard, Bauer \& Canguilhem [et al. ] 1958, p. 468.

91. Hamilton redéfinit l'action comme produit d'une énergie par un temps. 
émanations lointaines du principe de Héron ${ }^{92}$. Ces deux principes, régissant l'un la lumière, et l'autre la matière, ont longtemps été tenus pour indépendants, les physiciens ne voyant entre eux, de prime abord, qu'une analogie formelle sans base physique réelle. Ces principes ont même paru contradictoires, dans la mesure où la vitesse ne joue pas le même rôle dans l'un et dans l'autre. Alors que la vitesse figure au dénominateur dans le principe de Fermat (un temps est le rapport d'une distance à une vitesse), elle apparaît au numérateur chez Maupertuis (l'action est le produit d'une distance par une vitesse). Il faudra attendre le $\mathrm{XX}^{\mathrm{e}}$ siècle et Louis de Broglie pour que ces deux principes se trouvent conciliés dans la mécanique ondulatoire.

Seule la mécanique ondulatoire, écrit Louis de Broglie, en montrant qu'on doit associer au mouvement de tout point matériel la propagation d'une onde dont la vitesse de propagation varie en raison inverse de la vitesse du point matériel, a véritablement mis en lumière la parenté profonde des deux grands principes [de Fermat et Maupertuis], et la signification physique de cette parenté $e^{93}$.

On peut même tirer de l'identité des principes, élevée elle-même au rang de principe, la relation bien connue $: p=b / \lambda$, qui constitue un des fondements de la mécanique quantique. La Catoptrique de Héron nous conduit ainsi de manière assez inattendue aux portes de la physique la plus contemporaine.

\section{Signification épistémologique du principe de Héron}

Après ce parcours historique, destiné à montrer la postérité et la fécondité du principe de Héron, nous allons nous attacher à en dégager la signification épistémologique. Ce principe inscrit en effet le mécanicien dans une tradition scientifique bien particulière qui privilégie, au rebours de l'expérimentalisme et de l'instrumentalisme, la causalité finale et formelle dans l'explication des phénomènes et promeut les mathématiques au rang de principe ontologique fondamental.

\section{Le principe de Héron et la causalité finale}

La première caractéristique du principe de Héron, d'un point de vue épistémologique, est d'être un principe téléologique. Le rayon lumineux ou

92. Celui-ci ayant été transmis à Fermat par l'intermédiaire de Vitellion et de Cureau de la Chambre puis à Maupertuis par l'intermédiaire de Fermat lui-même. C'est en effet à l'occasion d'une polémique autour du principe de Fermat que Maupertuis a été mis sur la voie de son propre principe.

93. Broglie 1974, p. 37. 
plutôt visuel va d'un point à un autre de façon à rendre minimale la distance entre deux points. Il est sous-tendu par une thèse que l'on peut dire métaphysique, selon laquelle « la nature ne fait rien en vain ». Cette proposition ne se rencontre pas chez Héron, mais chez ses doxographes. Ainsi, selon le témoignage de Damien dans les Hypothèses optiques, Héron dit que « puisque la nature ne veut pas imposer au rayon visuel un chemin inutile, elle le brise suivant des angles égaux $\gg{ }^{94}$. De la même façon, Olympiodore affirme, dans son Commentaire des Météorologiques, que «puisqu'il est admis par tous que la nature ne fait rien en vain et ne s'efforce pas inutilement, si on nie que la réflexion a lieu suivant des angles égaux, la nature s'efforce inutilement en suivant des angles inégaux $\gg^{95}$. De ce point de vue, la démonstration héronienne de la réflexion se distingue fondamentalement de celle donnée par Ptolémée. Ptolémée ne fait pas intervenir la causalité finale, mais la causalité efficiente. Il déduit la loi en considérant la déviation que le miroir inflige au rayon visuel. La lumière est réfléchie parce que son mouvement est dévié par le miroir. Un miroir s'oppose à la pénétration des rayons visuels perpendiculaires, mais ne fait nullement obstacle aux rayons visuels tangents, qui continuent leur chemin sans être infléchis. Pour déterminer la loi, Ptolémée analyse en quelque sorte l'action ou plutôt la réaction du miroir sur le rayon visuel, l'effet qu'il produit sur son mouvement.

On peut éclairer la nature de cette opposition à l'aide de la distinction que l'on établit aujourd'hui entre deux grandes familles de principes physiques : les principes intégraux ou variationnels d'une part et les principes différentiels d'autre part. Le principe de Héron peut être considéré comme un principe intégral avant la lettre. Il énonce une condition portant sur la somme des déplacements élémentaires ou infinitésimaux du rayon visuel depuis son point de départ jusqu'à son point d'arrivée. L'intégrale des déplacements élémentaires calculée d'un point à l'autre doit être minimale, sa variation nulle. Ptolémée, en revanche, qui privilégie la causalité efficiente, adopte un point de vue différentiel. Il ne s'intéresse pas à la somme, mais à la série ou plutôt à la succession des déplacements élémentaires, le rayon visuel apprenant à chaque instant, en quelque sorte, ce qu'il doit faire à l'instant suivant. Il s'intéresse plus spécifiquement à ce qui se passe en un lieu singulier, celui où le rayon visuel rencontre l'obstacle constitué par le miroir. Pour dire les choses autrement, Héron envisage le phénomène globalement, Ptolémée l'appréhende localement. Héron ne suit pas la propagation ou la progression du rayon visuel de proche en proche ou d'instant en 
instant, mais considère l'ensemble du mouvement depuis son point de départ jusqu'à son point d'arrivée. Alors que Ptolémée accompagne le rayon visuel tout au long de son parcours et déduit le point d'arrivée du trajet suivi depuis le point de départ, ce qui paraît après tout bien naturel, notre mécanicien reconstruit, d'une manière assez paradoxale, la totalité du trajet à partir de la donnée des seuls points de départ et d'arrivée.

\section{L'acte et la puissance}

Le principe de Héron, qui, comme on vient de le voir est sous-tendu par une thèse métaphysique, ne révèle sa véritable originalité et sa signification profonde que replacé dans le cadre d'une distinction métaphysique, celle de la puissance et de l'acte, ou du possible et du réel. Héron admettrait, un peu comme Leibniz, que le réel n'est pas tout le possible, et qu'il y a des possibles qui ne se réaliseront pas. D'un point de vue logique, tous les chemins reliant le point de départ au point d'arrivée sont possibles, mais cette possibilité reste, en tant que telle, purement théorique. Tous ces chemins sont sans doute concevables sans contradiction, mais un seul se trouvera effectivement réalisé, celui qui minimise la distance. Le principe de Héron, comme tous les principes variationnels du reste, fixe en quelque sorte les conditions du passage du possible au réel, de la puissance à l'acte. Il détermine quel est, parmi tous les possibles, celui qui peut passer à l'existence et être réalisé par la Nature. On peut, sur ce point encore, mettre Héron en opposition avec Ptolémée. Ptolémée fonde la loi de la réfexion non pas sur un principe d'extrémalité, mais sur un principe de conservation, la conservation du mouvement. Or, selon la juste remarque de Jean Largeault: «Les principes de conservation et les principes de stationarité [comme celui de Héron] n'ont pas la même portée métaphysique: les premiers identifient des essences ou emploient des essences à identifier des systèmes au cours du temps ou du mouvement : les seconds individualisent $\gg^{96}$. Le principe de Héron est un principe d'individuation, l'individuation se faisant par la forme, et non par la matière. La forme (le plus court chemin) détermine l'existence physique. Là où Héron légitime une existence, Ptolémée identifie une essence, le mouvement. Pour Ptolémée, l'existence individuelle reste, dans le fond, inintelligible. Elle dépend de la condition initiale, qui n'est pas rationnelle. L'opposition entre Héron et Ptolémée est au fond la même que celle qu'on retrouvera à l'époque moderne entre Fermat et Descartes, ou encore entre Lagrange-Hamilton et Newton, les premiers mettant en avant des principes finaux, intégraux et globaux, les seconds des principes causaux (au sens de la causalité efficiente), différentiels et locaux. 


\section{L'idée d'une catoptrique rationnelle}

Aussi surprenant que cela paraisse, les deux familles de principes que nous venons de distinguer ne sont pas exclusives l'une de l'autre. Loin de se contredire et d'être mutuellement incompatibles, les principes différentiels et variationnels permettent même d'établir les mêmes lois. Leibniz a souligné à plusieurs reprises, et notamment dans son Discours de métaphysique, cette concordance, où il voit l'expression de l'harmonie préétablie entre le règne des causes finales et celui des causes efficientes :

Je trouve $[. .$.$] que plusieurs effets de la nature se peuvent démontrer dou-$ blement, savoir par la considération de la cause efficiente, et encore à part par la considération de la cause finale, en se servant par exemple du décret de Dieu de produire toujours son effet par les voies les plus aisées et les plus déterminées, comme j'ai fait voir ailleurs en rendant raison des règles de la catoptrique et de la dioptrique ${ }^{97}$.

J'ai coutume de dire, ajoute-t-il ailleurs, qu'il y a, pour parler ainsi, deux Règnes dans la nature corporelle même qui se pénètrent sans se confondre et sans s'empêcher: le Règne de la puissance, suivant lequel tout se peut expliquer mécaniquement par les causes efficientes, lorsque nous en pénétrons assez l'intérieur ; et aussi le Règne de la sagesse, suivant lequel tout se peut expliquer architectoniquement, pour ainsi dire, par les causes finales, lorsque nous en connaissons assez les usages ${ }^{98}$.

L'harmonie de ces deux attributs, déclare pour sa part Maupertuis, est si parfaite que tous les effets de la Nature se pourraient déduire de chacun pris séparément. Une mécanique aveugle et nécessaire suit les dessins de l'intelligence la plus éclairée et la plus libre ${ }^{99}$.

La démonstration mathématique de cette équivalence sera apportée, dans le domaine de la mécanique, par Lagrange puis par Hamilton qui déduiront les équations du mouvement (équations différentielles) du principe de moindre action (principe variationnel).

Bien qu'ils permettent d'établir les mêmes résultats, les principes variationnels n'obéissent pas en réalité aux mêmes motivations que les principes différentiels. Non seulement parce qu'ils sont plus propres à « purger,

97. Discours de métaphysique, $\$ 21$.

98. Tentamen anagogicum, dans Gerhardt 1890, p. 273. Cf. aussi Monadologie, §79-80.

99. Cité par Largeault 1985, p. 30. « Comme la construction du monde, déclare dans le même sens le mathématicien Euler, est la plus parfaite possible et qu'elle est due à un créateur infiniment sage, il n'arrive rien dans le monde qui ne présente des propriétés de maximum ou de minimum. C'est pourquoi aucun doute ne peut subsister sur ce qu'il soit également possible de déterminer tous les effets de l'univers par leurs causes finales, à l'aide de la méthode des maxima et des minima, aussi bien que par leurs causes effcientes. » (Euler 1744, p. 245.) 
comme dit Leibniz, la philosophie mécanique de la profanité qu'on lui impute $\gg{ }^{100}$, mais parce que leur ambition est fondamentalement différente. Alors que les principes différentiels sont orientés vers la prédiction et l'action, les principes variationnels privilégient au contraire la compréhension des phénomènes étudiés. Si les premiers anticipent ce qui sera à partir de ce qui est, les seconds s'attachent à rendre raison de ce qui a été. Ils ne décrivent pas un processus en train de se faire, mais rationalisent le phénomène tel qu'il s'est produit. La raison qu'ils apportent ne relève pas de la raison identitaire, qui réduit l'effet à sa cause, mais est de l'ordre du

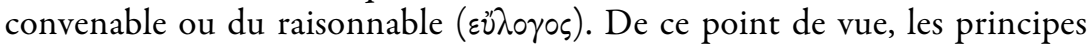
variationnels ne se rattachent pas, pour reprendre là encore une distinction leibnizienne, au principe de la nécessité ou de l'identité, mais à celui de la raison suffisante ou de la convenance ${ }^{101}$. Ces principes sont sans doute déterministes, mais leur déterminisme est moins rigide que le déterminisme aveugle du mécanisme. Cette rationalité singulière, plus souple mais tout aussi rigoureuse que la précédente, semble avoir été aperçue par Héron luimême, lorsqu'il indique que le rayon visuel, en suivant le plus court chemin, se réfléchit d'une manière conforme à la raison (rationabiliter). On pourrait dire, de ce point de vue, que notre mécanicien a été le promoteur de ce qu'on pourrait appeler «la catoptrique rationnelle », au même titre que Lagrange le sera plus tard de la mécanique rationnelle.

\section{Les mathématiques et le réel}

L'évocation du principe de la convenance ou du meilleur ne doit pas nous amener à penser que le principe de Héron serait d'essence secrètement théologique. Ce principe n'est pas théologique, mais mathématique et géométrique. Il est géométrique non seulement parce qu'il utilise des notions de géométrie (droite), ou parce que la démonstration de la loi fait intervenir des théorèmes de la géométrie euclidienne, mais parce que la géométrie se voit élevée au rôle de principe de la réalité physique elle-même. Héron déduit un phénomène physique (le trajet effectivement suivi par le rayon visuel) d'une contrainte géométrique (suivre le plus court chemin). Il ne faut pas se méprendre, en effet, sur la portée du principe. Héron ne veut pas dire simplement que la réflexion « isogonale » possède une propriété

100. Discours de métaphysique, $\$ 23$.

101. « Par la seule considération des causes efficientes ou de la matière, on ne saurait rendre raison, dit Leibniz, de ces lois du mouvement découvertes de notre temps et dont une partie a été découverte par moi-même. Car j'ai trouvé qu'il y faut recourir aux causes finales, et que ces lois ne dépendent point du principe de la nécessité comme les vérités logiques, arithmétiques et géométriques, mais du principe de la convenance, c'est-à-dire du choix de la sagesse. » (Principes de la nature et de la grâce, $\$ 11$.) 
parmi d'autres qui serait de correspondre au trajet le plus court, mais que la réflexion se fait à angles égaux pour minimiser le trajet. Autrement dit la condition de minimum n'est pas un accident, mais le principe et la fin de la réflexion elle-même. Héron ne le dit sans doute pas aussi clairement, mais c'est bien ainsi, en tout cas, que ses successeurs l'ont compris. Tout se passe, en d'autres termes, comme si la géométrie contraignait la réalité physique ou sensible. C'est là encore une idée profondément moderne. Elle se retrouve dans la théorie de la relativité d'Einstein, ou encore dans les théories de jauge en physique des particules. On peut sur ce point aussi mettre Héron en opposition avec Ptolémée, qui fait dans son astronomie, sinon dans son optique, un usage instrumental de la géométrie. Sa théorie des cycles et des épicycles n'est-elle pas autre chose, en effet, qu'un habile moyen de sauver les apparences?

Les principes téléologiques ont souvent intrigué les philosophes et les scientifiques, surtout à l'époque moderne. Comment, par exemple, la lumière peut-elle connaître le chemin le plus court pour aller d'un point à un autre ? Les positivistes, et notamment Mach, les ont ramenés à des artifices de calcul en dénonçant leur arrière-plan théologique et métaphysique. Mais il s'agit là d'une interprétation réductrice et simplificatrice, qui équivaut à les vider de leur contenu propre. Ces principes ne manifestent certes pas l'existence d'une providence mais un ordre mathématique immanent au réel lui-même. Cela veut dire que les mathématiques ne sont pas un simple langage, mais sont incarnées dans la nature. De ce point de vue, en dépit de l'usage qu'il fait de l'adage : la nature ne fait rien en vain, notre mécanicien serait au fond plus platonicien qu'aristotélicien. Il l'est non seulement parce qu'il reprend à son compte la théorie platonicienne de la vision, mais parce qu'il promeut, à travers son principe, la géométrie au rang de principe de la réalité physique. La nature est fin ou forme, mais cette fin ou cette forme sont mathématiques ou géométriques. Ce platonisme ou ce géométrisme ne nous renvoie pas à un autre âge de la science, mais peut être repéré jusque dans ses développements les plus récents.

En restant dans le domaine du monde «inorganique », écrit le mathématicien Maurice Janet, et sur un terrain purement positif, la science contemporaine retrouve, sous un nouvel aspect, l'idée de finalité. C'est une finalité dépouillée des idées anthropomorphiques et théologiques qui se sont trouvées si souvent associées à ce mot. Mais c'est une idée génératrice d'ordre et d'organisation : les trajectoires de la Dynamique se coordonnent, les vibrations propres apparaissent avec toutes leurs propriétés, les solutions des 
équations de la Physique mathématique manifestent lumineusement leur existence et laissent entrevoir la solidarité intime de toutes leurs parties ${ }^{102}$.

Cette idée d'une finalité purement géométrique de la Nature semble avoir été formulée pour la première fois - dans un contexte scientifique par Héron, dans un des chapitres de sa Catoptrique, qui s'avère de ce point de vue l'un des textes les plus emblématiques de l'Antiquité. 


\section{BIBLIOGRAPHIE}

Allard, G., E. BAuer, G. CANGuilhem [et al.] 1958 : Histoire générale des sciences, sous la direction de René Taton. 2, La science moderne, de 1450 à 1800, Paris, 1958.

BARTHOLIN, E. 1669 : Erasmi Bartholini experimenta crystalli islandici disdiaclastici, quibus mira et insolita refractio detegitur, Copenhague, 1669.

BRoglie, L. DE 1974 : La Physique nouvelle et les quanta, Paris, ${ }^{2} 1974$ (Nouvelle bibliothèque scientifique).

BRUnet, P. \& A. Mieli 1935: Histoire des sciences: Antiquité, Paris, 1935 (Bibliothèque scientifique).

Drachman, A. G. 1981 : « Hero of Alexandria », dans C. C. Gillispie (éd.), Dictionary of Scientific Biography. 6, New York, 1970, p. 310-314.

Chambry, É. 1933 : Platon, CEuvres complètes. 7. 1, La République : livres IV-VII, texte établi et traduit par - , Paris, 1933 (Collection des Universités de France).

Cureau de la Chambre, M. 1657 : La lumière [...], Paris, 1657.

EULER, L. 1744 : Methodus inveniendi lineas curvas maximi minimive proprietate gaudentes, sive Solutio problematis isoperimetrici latissimo sensu accepti, Lausanne-Genève, 1744.

FeYNMAN, R., R. R. LEIGHTON \& M. L. SANDS 1979 : Le cours de physique de Feynman. 1. 2, traitant surtout de la mécanique, du rayonnement et de la chaleur, Paris, 1979. [Traduction française, par G. Delacôte, de The Feynman Lectures on Physics.]

GERHARDT, C. I. (éd.) 1890 : Die philosophischen Schriften von Gottfried Wilhelm Leibniz, 7, Berlin, 1890.

Heath, Th. L. 1921 : A History of Greek Mathematics. 2, From Aristarchus to Diophantus, Oxford, 1921.

HeIBERG, J. L. 1925 : Geschichte der Mathematik und der Naturwissenschaften im Altertum (= Handbuch der Altertumswissenschaft, begr. von Iwan von Müller. V.1.2), Munich, 1925.

HiLl, D. R. 1988 : Héron d'Alexandrie, Les Mécaniques ou L'Élévateur des corps lourds, texte arabe de Qusta Ibn Luqa établi et trad. par B. Carra de Vaux ; introd. par D. R. Hill ; commentaires par A. G. Drachman, Paris, 1988 (Sciences et philosophie arabes. Études et reprises, 4).

Hultsch, Fr. 1876-1878 : Pappi Alexandrini Collectionis quae supersunt e libris manu scriptis edidit, latina interpretatione et commentariis instruxit - , Berlin, 3 vol., 1876-1878.

ITARD, J. 1966 : Mathématiques pures et appliquées, dans R. Arnaldez, J. Beaujeu, R. Bloch [et al.], Histoire générale des sciences, sous la direction de René Taton. Vol. 1, La science antique et médiévale : des origines à 1450, Paris, ${ }^{2} 1966$, p. 321-354.

JANET, M. 1932-1933 : «La finalité en mathématique et en physique », Recherches philosophiques, 2 (1932-1933), p. 1-17.

JANET, P. 1882 : Les causes finales, Paris, ${ }^{2} 1882$.

LaRgeault, J. 1985 : Principes de philosophie réaliste, Paris, 1985 (Philosophia, 10).

- 1988: Principes classiques d'interprétation de la nature, Paris-Lyon, 1988 (Science, histoire, philosophie).

Lejeune, A. 1956: L'Optique de Claude Ptolémée, dans la version latine d'après l'arabe de l'émir Eugène de Sicile, édition critique et exégétique, Louvain, 1956 (Université de Louvain. Recueil de travaux d'histoire et de philologie, $4^{\mathrm{c}}$ série, fasc. 8 ).

- 1957 : Recherches sur la catoptrique grecque d'après les sources antiques et médiévales, Bruxelles, 1957 (Académie Royale de Belgique, Mémoires de la Classe des Lettres et des Sciences morales et politiques, $2^{\mathrm{c}}$ série, t. $52.2=\mathrm{n}^{\circ} 1676$ ). 
LLOYD, G. E. R. 1993 : Une Histoire de la science grecque [traduction par J. Brunschwig de : Early Greek Science $=$ Les Débuts de la science grecque et de: Greek Science after Aristotle = La Science grecque après Aristote], Paris, 1993 (Points. Sciences, 92).

MARTIN, TH. H. 1854 : Recherches sur la vie et les ouvrages d'Héron d'Alexandrie, disciple de Ctésibius, et sur tous les ouvrages mathématiques grecs, conservés ou perdus, publiés ou inédits, qui ont été attribués à un auteur nommé Héron, Paris, 1854 (Mémoires présentés par divers savants à l'Académie des inscriptions et belles-lettres de l'Institut de France. Première série, Sujets divers d'érudition, 4).

- 1871 : «Ptolémée auteur de l'Optique traduite en latin par Ammiratus Eugenius Siculus sur une traduction arabe incomplète, est-il le même que Claude Ptolémée, auteur de l'Almageste ? », Bulletino di bibliografia e di storia delle scienze matematiche e fisiche, 4 (novembre 1871).

Maupertuis, P. L. MoreaU De 1753 : Les auvres de Mr de Maupertuis. 1, Berlin-Lyon, 1753.

Neugebauer, O. 1938 : Ueber eine Methode zur Distanzbestimmung : Alexandria-Rom bei Heron, Copenhague, 1938 (Kongelige danske videnskabernes selskab. Historiskfilologiske meddelelser, 26.2).

PeyrouX, J. 1985 : Godefroy-Guillaume Leibnitz, CEuvre concernant la physique, trad. pour la première fois du latin en français, avec des notes, [Bordeaux]-Paris, 1985.

RISNER, FR. 1572 : Opticae thesaurus: Alhazeni Arabis [Opticae] libri septem nunc primum editi; ejusdem Liber de crepusculis et nubium ascensionibus. Item Vitellonis Thuringopoloni [Opticae] libri decem, omnes instaurati, figuris illustrati \& aucti, adjectis etiam in Alhazenum commentariis, Bâle, 1572.

Rivaud, A. 1925 : Platon, CEuvres complètes. 10, Timée; Critias, texte établi et traduit par -, Paris, 1925 (Collection des Universités de France).

Robin, L. 1926 : Platon, CEuvres complètes. 4.1, Phédon, texte établi et traduit par -, Paris, 1926 (Collection des Universités de France).

SCHMIDT, W., L. NIX \& J.L. HeIBERG 1899-1914: Heronis Alexandrini Opera que supersunt omnia, Stuttgart, 5 vol., 1899-1914 (Bibliotheca scriptorum graecorum et romanorum teubneriana).

SCHÖNE, R. 1897 : Damianos Schrift über Optik, mit Auszügen aus Geminos, griechisch und deutsch herausgegeben von -, Berlin, 1897.

Serres, M. 1984: Pierre-Simon Laplace, Exposition du système du monde, [Paris], 1984 ( Corpus des œuvres de philosophie en langue française).

SimON, G. 1988 : Le regard, l'être et l'apparence dans l'optique de l'Antiquité, Paris,1988 (Des Travaux).

STÜVE, W. 1900 : Olympiodori in Aristotelis Meteora commentaria, edidit —, Berlin, 1900 (Commentaria in Aristotelem Graeca. 12.2).

Tannery, P. \& Ch. Henry 1894: QEuvres de Fermat, publ. par les soins de - . 2, Correspondance, Paris, 1894.

Tannery, P. \& CH. Henry 1896: CEuvres de Fermat, publ. par les soins de - . 3, Traductions par P. Tannery $1^{\circ}$ ) Des écrits et fragments latins de Fermat. $\left.2^{\circ}\right)$ De l'Inventum novum de Jacques de Billy. $3^{\circ}$ ) Du Commercium epistolicum de Wallis, Paris, 1896.

Ver EeCKe, P. 1938 : Euclide, L'Optique et la Catoptrique, œuvres traduites pour la première fois du grec en français avec une introduction et des notes, Paris-Bruges, 1938. 